\title{
Equivariant cohomology distinguishes geometric structures of toric hyperKahler manifolds
}

\section{Shintaro Kuroki}

\begin{tabular}{|c|l|}
\hline Citation & OCAMI Preprint Series \\
\hline Issue Date & 2010 \\
\hline Type & Preprint \\
\hline Textversion & Author \\
\hline Rights & For personal use only. No other uses without permission. \\
\hline Relation & $\begin{array}{l}\text { This is a pre-print of an article published in Proceedings of the } \\
\text { Steklov Institute of Mathematics. The final authenticated } \\
\text { version is available online at: } \\
\text { https://doi.org/10.1134/S0081543811080189 }\end{array}$ \\
\hline
\end{tabular}

From: Osaka City University Advanced Mathematical Institute

http://www.sci.osaka-cu.ac.jp/OCAMI/publication/preprint/preprint.html 


\title{
Equivariant cohomology distinguishes geometric structures of toric hyperKähler manifolds
}

\author{
Shintarô Kuroki
}

\begin{abstract}
Toric hyperKähler manifolds are the hyperKähler analogue of symplectic toric manifolds. The purpose of this paper is to study some variation of cohomological rigidity theorem for toric hyperKähler manifolds. Roughly, we prove that the weak $H^{*}(B T)$-algebra structure of equivariant cohomology determines the weak $T$-hyperhamiltonian structure of toric hyperKähler manifolds.
\end{abstract}

\section{Introduction}

A toric hyperKähler manifold is defined by the hyperKähler quotient of a torus action on the quaternionic space $\mathbb{H}^{m}$. This space is introduced as the hyperKähler analogue of symplectic toric manifolds in [BiDa, Go]. The resulting manifolds are $4 n$-dimensional manifolds with quarter dimensional torus actions, i.e., with $T^{n}$-actions (see Section 2). We note that symplectic toric manifolds are $2 n$-dimensional manifolds with half dimensional torus actions. So, as a space, symplectic toric manifolds and toric hyperKähler manifolds are quite different. However, in [BiDa], Bielawski-Dancer show that there is a one-to-one correspondence between toric hypreKähler manifolds (geometry) and smooth hyperplane arrangements (combinatorics) (see Section 3). This is the similar phenomena to that toric manifolds can be described by using the combinatorial objects, fans or polytopes (see [BuPa, Fu, Od] for detail). Namely, as far as toric hyperKähler manifolds (or toric manifolds) are considered, the following two informations are the same:

$$
\text { Geometry } \Longleftrightarrow \text { Combinatorics }
$$

In particular, using this correspondence, equivariant cohomologies of toric manifolds and toric hyperKähler manifolds can be computed (see [Fu, Od] for toric manifolds and [Ko1, Ko2] for toric hyperKähler manifolds). Here, the equivariant cohomology is the important invariant of group actions (see $[\mathbf{B r}, \mathbf{H s}, \mathbf{K a}]$ for detail, and also see Section 5). Furthermore, in the case of toric manifolds, the following important theorem are proved by Masuda in [Ma2]:

TheOREM 1 (Masuda). Two toric manifolds are isomorphic as varieties if and only if their equivariant cohomology algebras are weakly isomorphic.

The Masuda's theorem means that the informations coming from the equivariant cohomology (algebra) has also the same informations with those coming from geometry and combinatorics,

2000 Mathematics Subject Classification. Principal: 55N91; 57S25, Secondly: 14M25; 32S22; 37J05.

Key words and phrases. Cohomological rigidity, Equivariant cohomology, Hyperhamiltonian isometry, Hyperplane arrangement, Toric hyperKähler manifold (hypertoric manifold).

The author was supported in part by Basic Science Research Program through the NRF of Korea funded by the Ministry of Education, Science and Technology (2010-0001651) and the Fujyukai Foundation. 
i.e., we have the following trinity for the toric manifolds:

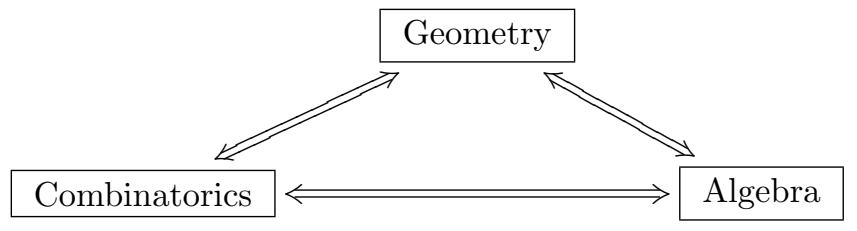

Motivated by the Masuda's theorem, the purpose of this paper is to show the trinity above also holds for toric hyperKähler manifolds. Namely, the goal of this paper is to prove the following theorem (see Theorem 4.4 and 8.1 for detail):

THEOREM 1.1. Let $\left(M_{\alpha}, T, \mu_{\widehat{\alpha}}\right)$ and $\left(M_{\beta}, T, \mu_{\widehat{\beta}}\right)$ be toric hyperKähler manifolds $M_{\alpha}, M_{\beta}$ with hyperKähler moment maps $\mu_{\widehat{\alpha}}, \mu_{\widehat{\beta}}$, and $\mathcal{H}_{\widehat{\alpha}}, \mathcal{H}_{\widehat{\beta}}$ be their hyperplane arrangements, respectively. Then, the following three statements are equivalent:

(1) $\left(M_{\alpha}, T, \mu_{\widehat{\alpha}}\right) \equiv_{w}\left(M_{\beta}, T, \mu_{\widehat{\beta}}\right)$;

(2) $\mathcal{H}_{\widehat{\alpha}} \equiv_{w} \mathcal{H}_{\widehat{\beta}}$;

(3) there exists a weak $H^{*}(B T)$-algebra isomorphism $f_{T}^{*}: H_{T}^{*}\left(M_{\alpha} ; \mathbb{Z}\right) \rightarrow H_{T}^{*}\left(M_{\beta} ; \mathbb{Z}\right)$ such that $\left(f_{T}^{*}\right)_{\mathbb{R}}(\widehat{a})=\widehat{b}$,

where $\widehat{a}=J_{m_{1}}^{*}(\widehat{\alpha}) \in H_{T}^{2}\left(M_{\alpha} ; \mathbb{R}\right), \widehat{b}=J_{m_{2}}^{*}(\widehat{\beta}) \in H_{T}^{2}\left(M_{\beta} ; \mathbb{R}\right)$ and $\left(f_{T}^{*}\right)_{\mathbb{R}}: H_{T}^{*}\left(M_{\alpha} ; \mathbb{R}\right) \rightarrow H_{T}^{*}\left(M_{\beta} ; \mathbb{R}\right)$ is the natural isomorphism induced from $f_{T}^{*}$.

Here, in Theorem 1.1, three equivalence relations are introduced in Section 4.1 for toric hyperKähler manifolds, Section 4.2 for hyperplane arrangements, and Section 5.1 for equivariant cohomologies; and $J_{m_{1}}:\left(\mathfrak{t}^{m_{1}}\right)^{*} \rightarrow H_{T}^{2}\left(M_{\alpha} ; \mathbb{R}\right)$ and $J_{m_{2}}:\left(\mathfrak{t}^{m_{2}}\right)^{*} \rightarrow H_{T}^{2}\left(M_{\beta} ; \mathbb{R}\right)$ are isomorphisms defined in Section 7.1.2, where $M_{\alpha}, M_{\beta}$ are defined by the hyperKähler quotient on $\mathbb{H}^{m_{1}}, \mathbb{H}^{m_{2}}$, respectively. In this paper, $H^{*}(X)$ always represents $H^{*}(X ; \mathbb{Z})$.

Theorem 1.1 tells us that, as far as toric hyperKähler manifolds are considered, we have a trinity among toric hyperKähler manifolds with hyperKähler moment maps $\left(M_{\alpha}, T, \mu_{\widehat{\alpha}}\right)$ (geometry), smooth hyperplane arrangements $\mathcal{H}_{\widehat{\alpha}} \subset\left(\mathfrak{t}^{n}\right)^{*}$ (combinatorics), and equivariant cohomologies with non-zero element $\widehat{a} \in H_{T}^{2}\left(M_{\alpha} ; \mathbb{R}\right)\left(H_{T}^{*}\left(M_{\alpha}\right), \pi^{*}, \widehat{a}\right)$ (algebra) such as the following diagram:

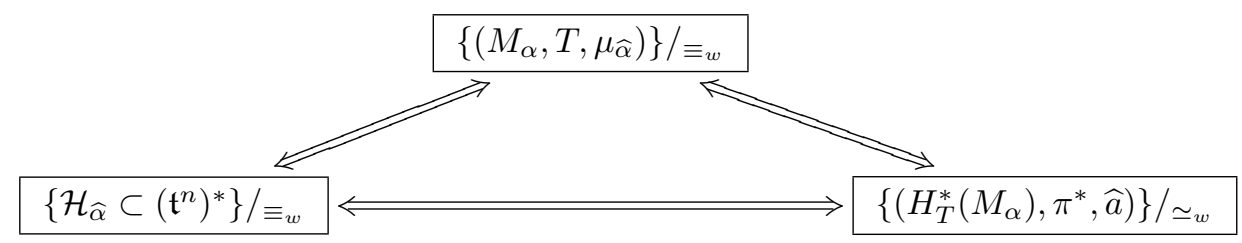

The organization of this paper and the idea of proof of Theorem 1.1 are as follows. We first recall toric hyperKähler manifolds and their basic facts in Section 2, and we then introduce hyperplane arrangements defined by toric hyperKähler manifolds in Section 3. In Section 4, we introduce two equivalence relations on toric hyperKähler manifolds and hyperplane arrangements, and prove these two equivalence relations are compatible (in Theorem 4.4). In Section 5, we recall the basic facts about equivariant cohomologies of toric hyperKähler manifolds. The idea of proof of Theorem 1.1 is to translate the original hyperplane arrangements defined in the dual of Lie algebra $\left(\mathfrak{t}^{n}\right)^{*}$ into the hyperplane arrangements defined in the equivariant cohomology $H^{2}\left(B T^{n} ; \mathbb{R}\right)$. So, in Section 6 , we define the hyperplane arrangement in $H^{2}\left(B T^{n} ; \mathbb{R}\right)$, and in Section 7 , we prove two hyperplane arrangements in $\left(\mathfrak{t}^{n}\right)^{*}$ and $H^{2}\left(B T^{n} ; \mathbb{R}\right)$ are equivalent up to weak equivalence defined in Section 4 (in Proposition 7.11). Finally, in Section 8, we prove a weak $H^{*}(B T)$-algebra isomorphism between two equivariant cohomologies $H_{T}^{*}\left(M_{\alpha}\right)$ and $H_{T}^{*}\left(M_{\beta}\right)$ induces a weak equivalence between two hyperplane arrangements in $H^{*}(B T ; \mathbb{R}) \stackrel{\pi_{3}^{*}}{\rightarrow} H_{T}^{*}\left(M_{\alpha} ; \mathbb{R}\right)$ and $H^{*}(B T ; \mathbb{R}) \stackrel{\pi_{2}^{*}}{\rightarrow} H_{T}^{*}\left(M_{\beta} ; \mathbb{R}\right)$. This yields the trinity described as above. 


\section{Toric hyperKähler varieties}

First of all, in this section, we recall the basic facts of the toric hyperKähler variety needed later (see [BiDa, HaSt, Ko3, Pr], for detail). We assume throughout this paper that $\mathbb{Z}$ is the integer, $\mathbb{R}$ is the real, $\mathbb{C}$ is the complex and $\mathbb{H}$ is the quaternionic numbers, i.e., $\mathbb{H} \simeq \mathbb{R}^{4}$ as the $\mathbb{R}$-vector space whose basis are $1, \mathbf{i}, \mathbf{j}, \mathbf{k}$ and they satisfy the following quaternionic relations:

$$
\mathbf{i j k}=\mathbf{i}^{2}=\mathbf{j}^{2}=\mathbf{k}^{2}=-1 .
$$

2.1. Definition of toric hyperKähler manifolds and their geometric structures. We first define a toric hyperKähler manifold and introduce its geometric structure.

2.1.1. HyperKähler structure on $\mathbb{H}^{m}$. In the beginning, let us recall a geometric structure on the quaternionic vector space. Assume $\mathbb{H}^{m}$ is the $m$-dimensional quaternionic vector space with the left $\mathbb{H}$-scalar product. Then the complex structure $I_{1}: \mathbb{H}^{m} \rightarrow \mathbb{H}^{m}$ with $I_{1}^{2}=-\mathrm{Id}_{\mathbb{H}^{m}}$ (resp. $I_{2}$ and $I_{3}$ ) on $\mathbb{H}^{m}$ is defined by the left multiplication of $\mathbf{i}$ (resp. $\mathbf{j}$ and $\mathbf{k}$ ). We now put the flat Riemannian metric $g$ on $\mathbb{H}^{m}$ arising from the standard Euclidean scaler product on $\mathbb{H}^{m} \simeq \mathbb{R}^{4 m}=\mathbb{R}^{m} \oplus \mathbf{i} \mathbb{R}^{m} \oplus \mathbf{j} \mathbb{R}^{m} \oplus \mathbf{k} \mathbb{R}^{m}$. Using these structures, we can define three Kähler forms on $\mathbb{H}^{m}$ as follows:

$$
\omega_{i}(X, Y)=g\left(I_{i} X, Y\right) \quad i=1,2,3
$$

where $X, Y$ are tangent vectors on a point in $\mathbb{H}^{m}$. The metric $g$ is a hyperKähler metric, i.e., it is a Kähler metric with respect to all three complex structures $I_{1}, I_{2}$ and $I_{3}$ which satisfy the quaternionic relations. The automorphism group which preserves the hyperKähler structure (i.e., $g, I_{1}, I_{2}$ and $\left.I_{3}\right)$ is called the symplectic group and denoted by $S p(m)$, i.e., the subgroup of special orthogonal group $S O(4 m)$ which commutes with $I_{1}, I_{2}$ and $I_{3}$, or equivalently preserves the Kähler forms $\omega_{1}, \omega_{2}$ and $\omega_{3}$. Note that $S p(m)$ acts on $\mathbb{H}^{m}$ from the right. In this paper, if the group $G$ acts on a hyperKähler manifold $M$ with preserving its hyperKähler structure, we call $G$ acts on $\left(M, g_{M}, I_{M}, J_{M}, K_{M}\right)$, where $g_{M}$ is a Riemannian structure and $I_{M}, J_{M}$ and $K_{M}$ are three complex structures which define the hyperKähler structure on $M$.

2.1.2. HyperKähler moment map of $\left(\mathbb{H}^{m}, T^{m}\right)$. We next recall properties of the torus action on $\left(\mathbb{H}^{m}, g, I_{1}, I_{2}, I_{3}\right)$. Because $S p(m)$ acts on $\left(\mathbb{H}^{m}, g, I_{1}, I_{2}, I_{3}\right)$, a maximal torus in $S p(m)$ preserves the hyperKähler structure on $\mathbb{H}^{m}$. Let $T^{m}$ be the diagonal abelian subgroup in $S p(m)$, i.e., $m$ dimensional torus. This torus $T^{m}$ is a maximal torus in $S p(m)$, and the $T^{m}$-action on $\mathbb{H}^{m}$ is defined by right multiplication:

$$
\begin{array}{ccc}
\mathbb{H}^{m} & \longrightarrow & \mathbb{H}^{m} \\
\Psi & \stackrel{\cdot}{\uplus} & \Psi \\
z+w \mathbf{k} & \stackrel{\cdot}{\longmapsto} & z t+w t^{-1} \mathbf{k}
\end{array}
$$

for $z, w \in \mathbb{C}^{m}$ and $t \in T^{m}$. By using this action, we can regard $\mathbb{H}^{m}$ as $T^{*} \mathbb{C}^{m}$, i.e., the cotangent bundle of $\mathbb{C}^{m}$; or equivalently $\mathbb{C}^{m} \oplus \overline{\mathbb{C}^{m}}$, where $\overline{\mathbb{C}^{m}}$ is isomorphic to $\mathbb{C}^{m}$ with reversed orientation.

Regard a symplectic form on $\mathbb{H}^{m}$ as $\omega_{\mathbb{R}}=\omega_{1}$ and a holomorphic symplectic form on $\mathbb{H}^{m}$ as $\omega_{\mathbb{C}}=\omega_{2}+\sqrt{-1} \omega_{3}$. Then the $T^{m}$-action defined in $(2.2)$ preserves $\omega_{\mathbb{R}}$ and $\omega_{\mathbb{C}}$, and induces the hyperKähler moment map

$$
\mu_{\mathbb{R}} \oplus \mu_{\mathbb{C}}: \mathbb{H}^{m} \longrightarrow\left(\mathfrak{t}^{m}\right)^{*} \oplus\left(\mathfrak{t}_{\mathbb{C}}^{m}\right)^{*}
$$

such that

$$
\mu_{\mathbb{R}}(z, w)=\frac{1}{2} \sum_{i=1}^{m}\left(\left|z_{i}\right|^{2}-\left|w_{i}\right|^{2}\right) \partial_{i}
$$

and

$$
\mu_{\mathbb{C}}(z, w)=2 \sqrt{-1} \sum_{i=1}^{m} z_{i} w_{i} \partial_{i},
$$

where $z=\left(z_{1}, \ldots, z_{m}\right) \in \mathbb{C}^{m}$ and $w=\left(w_{1}, \ldots, w_{m}\right) \in \overline{\mathbb{C}^{m}}$ for $\mathbb{H}^{m}=\mathbb{C}^{m} \oplus \overline{\mathbb{C}^{m}}$, and $\partial_{i}(i=$ $1, \ldots, m)$ is the basis in $\left(\mathfrak{t}^{m}\right)^{*}$ and $\left(\mathfrak{t}_{\mathbb{C}}^{m}\right)^{*}$. 
2.1.3. Definition of a toric hyperKähler variety. In order to define a toric hyperKähler variety, we explain the hyperKähler quotient.

Let $K$ be a connected subgroup $T^{m}$. There is the following sequence:

$$
K \stackrel{\iota}{\longrightarrow} T^{m} \stackrel{\rho}{\longrightarrow} T^{m} / K \simeq T^{n},
$$

where $\iota$ is the inclusion, $\rho$ is the projection to the cokernel of $\iota$, and put $n=m-\operatorname{dim} K$. This sequence induces the following exact sequence of Lie algebras:

$$
\{0\} \longrightarrow \mathfrak{k} \stackrel{\iota_{*}}{\longrightarrow} \mathfrak{t}^{m} \stackrel{\rho_{*}}{\longrightarrow} \mathfrak{t}^{n} \longrightarrow\{0\} .
$$

Taking its dual, we have the following exact sequence:

$$
\{0\} \longrightarrow\left(\mathfrak{t}^{n}\right)^{*} \stackrel{\rho^{*}}{\longrightarrow}\left(\mathfrak{t}^{m}\right)^{*} \stackrel{\iota^{*}}{\longrightarrow} \mathfrak{k}^{*} \longrightarrow\{0\} .
$$

By using $\iota^{*}$ and its complexification $\iota_{\mathbb{C}}^{*}$, we can define the hyperKähler moment map of $K$-action on $\mathbb{H}^{m}$ as follows:

$$
\mu_{H K}: \mathbb{H}^{m} \stackrel{\mu_{\mathbb{R}} \oplus \mu_{\mathbb{C}}}{\longrightarrow}\left(\mathfrak{t}^{m}\right)^{*} \oplus\left(\mathfrak{t}_{\mathbb{C}}^{m}\right)^{*} \stackrel{\iota^{*} \oplus \iota_{\mathbb{C}}^{*}}{\longrightarrow} \mathfrak{k}^{*} \oplus \mathfrak{k}_{\mathbb{C}}^{*} .
$$

By the definition of $\mu_{H K}$, an element $(\alpha, 0) \in \mathfrak{k}^{*} \oplus \mathfrak{k}_{\mathbb{C}}^{*}$ for each non-zero $\alpha \in \mathfrak{k}^{*}$ is a regular value of $\mu_{H K}$. Hence, its inverse image $\mu_{H K}^{-1}(\alpha, 0)$ has the almost free $K$-action. Therefore, its quotient space $\mu_{H K}^{-1}(\alpha, 0) / K$ is a $4 n$-dimensional orbifold with the induced $T^{m} / K\left(\simeq T^{n}\right)$ action from $T^{m}$-action on $\mu_{H K}^{-1}(\alpha, 0)$. This quotient is called the hyperKähler quotient.

Put

$$
M_{\alpha}=\mu_{H K}^{-1}(\alpha, 0) / K \text {. }
$$

We call $M_{\alpha}$ a toric hyperKähler variety. If $M_{\alpha}$ is non-singular, then we call it a toric hyperKähler manifold. The following proposition gives the necessary and sufficient condition for the smoothness of a toric hyperKähler variety (see [Ko1, Proposition 2.2]).

Proposition 2.1. The following two statements are equivalent.

(1) The action of $K$ on $\mu_{H K}^{-1}(\alpha, 0)$ is free, i.e., $M_{\alpha}=\mu_{H K}^{-1}(\alpha, 0) / K$ is a manifold.

(2) For any $J \subset\{1, \ldots, m\}$ such that $\left\{\iota^{*} u_{j} \mid j \in J\right\}$ forms a basis of $\mathfrak{k}^{*}$,

$$
\mathfrak{t}_{\mathbb{Z}}^{m}=\mathfrak{k}_{\mathbb{Z}} \oplus \sum_{j \in J^{c}} \mathbb{Z} \partial_{j} \quad \text { as a } \mathbb{Z} \text {-module, }
$$

where we regard $\mathfrak{k}$ as the subset of $\mathfrak{t}^{m}$ via $\iota_{*}, u_{j}$ is an element of $\left(\mathfrak{t}^{m}\right)^{*}$, and $\mathfrak{k}_{\mathbb{Z}}$ and $\mathfrak{t}_{\mathbb{Z}}^{m}$ are lattice subgroups of $\mathfrak{k}$ and $\mathfrak{t}^{m}$ respectively.

We also note the following proposition:

Proposition 2.2. Let $u_{m} \in\left(\mathfrak{t}^{m}\right)^{*}$ be a basis. If $\iota^{*}\left(u_{m}\right)=0$, then there exists the $(4 n-4)$ dimensional toric hyperKähler manifold $M$ such that

$$
\mu_{H K}^{-1}(\alpha, 0) / K=M \times \mathbb{H},
$$

where $M \times \mathbb{H}$ has the diagonal $T^{n-1} \times S^{1}$-action and the $S^{1}$-action on $\mathbb{H}$ is the standard action.

Proof. Because $u_{m}$ is a basis of $\left(\mathfrak{t}^{m}\right)^{*}$, there exists the $(m-1)$-dimensional subspace in $\mathfrak{t}^{m}$ defined by

$$
\left\{x \in \mathfrak{t}^{m} \mid\left\langle u_{m}, x\right\rangle=0\right\},
$$

where $\langle$,$\rangle is the natural paring of Lie algebra and its dual, i.e., \left\langle u_{m}, x\right\rangle=u_{m}(x)$. Denote this subspace by $\mathfrak{t}^{m-1}$.

Assume $\iota^{*}\left(u_{m}\right)=0$. Let $y \in \mathfrak{k}$. Then, we have

$$
\left\langle u_{m}, \iota_{*}(y)\right\rangle=\left\langle\iota^{*}\left(u_{m}\right), y\right\rangle=0 .
$$

Therefore, $\iota_{*}(\mathfrak{k}) \subset \mathfrak{t}^{m-1}$. In particular, taking their exponent, we have $K \subset T^{m-1}$. Hence, $\mu_{H K}^{-1}(\alpha, 0) / K$ may be regarded as the hyperKähler quotient of the diagonal action of $K \times\{e\}$ on $\mathbb{H}^{m-1} \times \mathbb{H}$. By the definition of hyperKähler quotient, the space appearing as the hyperKähler quotient of the identity group $\{e\}$-action on $\mathbb{H}$ is $\mathbb{H}$ itself. Therefore, for the hyperKähler quotient $M$ of $K$-action on $\mathbb{H}^{m-1}$, we have that $\mu_{H K}^{-1}(\alpha, 0) / K=M \times \mathbb{H}$. 
2.1.4. HyperKähler moment map of $\left(M_{\alpha}, T^{n}\right)$. By the definition of the toric hyperKähler variety $M_{\alpha}$, the $T^{n}$-action on the smooth part of $M_{\alpha}$ preserves three Kähler forms $\widetilde{\omega}_{i}$ induced from $\omega_{i}, i=1,2,3$ (see $(2.1)$ ), i.e., this action preserves the hyperKähler structure on the smooth part of $M_{\alpha}$. Hence, this $T^{n}$-action also preserves the real symplectic form $\widetilde{\omega}_{\mathbb{R}}=\widetilde{\omega}_{1}$ and the holomorphic symplectic form $\widetilde{\omega}_{\mathbb{C}}=\widetilde{\omega}_{2}+\sqrt{-1} \widetilde{\omega}_{3}$ on the smooth part of $M_{\alpha}$. Define a hyperKähler moment map $\mu_{\widehat{\alpha}}=\widetilde{\mu}_{\mathbb{R}} \oplus \widetilde{\mu}_{\mathbb{C}}$ as follows:

$$
\begin{aligned}
\widetilde{\mu}_{\mathbb{R}}[z, w] \oplus \widetilde{\mu}_{\mathbb{C}}[z, w] & =\left(\frac{1}{2} \sum_{i=1}^{m}\left(\left|z_{i}\right|^{2}-\left|w_{i}\right|^{2}\right)-\alpha_{i}\right) \partial_{i} \oplus 2 \sqrt{-1} \sum_{i=1}^{m} z_{i} w_{i} \partial_{i} \\
& \in \operatorname{ker} \iota^{*} \oplus \operatorname{ker} \iota_{\mathbb{C}}^{*} \simeq\left(\mathfrak{t}^{n}\right)^{*} \oplus\left(\mathfrak{t}_{\mathbb{C}}^{n}\right)^{*},
\end{aligned}
$$

where $[z, w] \in M_{\alpha}$ and $\alpha_{i}$ is the $i^{\text {th }}$ element of $\widehat{\alpha}=\left(\alpha_{1}, \ldots, \alpha_{m}\right) \in\left(\iota^{*}\right)^{-1}(\alpha) \subset\left(\mathfrak{t}^{m}\right)^{*}$; we call $\widehat{\alpha} \in\left(\mathfrak{t}^{m}\right)^{*}$ a lift of $\alpha \in\left(\mathfrak{t}^{n}\right)^{*}$.

We note the following remark (assumption) needed in Section 8.

REMARK 2.3. Suppose $\iota^{*}\left(u_{m}\right)=0$. Then, by Proposition 2.2, the toric hyperKähler manifold is $\mu_{H K}(\alpha, 0) / K=M \times \mathbb{H}$. Note that $S^{1}$ acts on $\mathbb{H}$ standardly. In this paper, we assume that the hyperKähler moment map of the standard $S^{1}$-action on $\mathbb{H}$ is always defined by the map $\mu=\mu_{\mathbb{R}} \oplus \mu_{\mathbb{C}}: \mathbb{H} \rightarrow \mathbb{R} \oplus \mathbb{C}$ in Section 2.1.2. Therefore, by the definition of the hyperKähler moment map $\mu_{\widehat{\alpha}}$, it is easy to check the following decomposition:

$$
\mu_{\widehat{\alpha}}=\mu_{\widehat{\alpha}^{\prime}} \oplus \mu,
$$

for some lift $\widehat{\alpha}^{\prime} \in\left(\mathfrak{t}^{m-1}\right)^{*}$ of $\alpha \in \mathfrak{k}^{*}$ (note that $K \subset T^{m-1}$ in this case). Namely, if $\iota^{*}\left(u_{m}\right)=0$ then we always assume a lift $\widehat{\alpha} \in\left(\mathfrak{t}^{m}\right)^{*}$ of $\alpha$ as follows:

$$
\left(\widehat{\alpha}^{\prime}, 0\right) \in\left(\mathfrak{t}^{m-1}\right)^{*} \oplus\left(\mathfrak{t}^{1}\right)^{*} .
$$

2.2. Example and Remark. Here, we give the standard example of toric hyperKähler varieties.

EXAMPLE 2.4. Let $\Delta$ be the diagonal subgroup in $T^{n+1}$. Then, we get the following exact sequence by using the inclusion $\Delta \subset T^{n+1}$ :

$$
\left(\mathfrak{t}^{n}\right)^{*} \stackrel{\rho^{*}}{\longrightarrow}\left(\mathfrak{t}^{n+1}\right)^{*} \stackrel{\iota^{*}}{\longrightarrow} \mathbb{R}
$$

such that

$$
\iota^{*}\left(\alpha_{1}, \ldots, \alpha_{n+1}\right)=\alpha_{1}+\cdots+\alpha_{n+1} \in \mathbb{R},
$$

where $\left(\alpha_{1}, \ldots, \alpha_{n+1}\right) \in\left(\mathfrak{t}^{n+1}\right)^{*} \simeq \mathbb{R}^{n+1}$ and $\mathbb{R}$ is the dual of Lie algebra of $\Delta$.

Put $\alpha=n+1 \in \mathbb{R}$. Then the toric hyperKähler manifold $\mu_{H K}^{-1}(\alpha, 0) / \Delta$ is $T^{n}$-equivariantly diffeomorphic to $T^{*} \mathbb{C} P^{n}$, where the $T^{n}$-action on $T^{*} \mathbb{C} P^{n}$ is induced from the standard $T^{n}$-action on $\mathbb{C} P^{n}$.

We finish this section by the following remark (assumption).

REMARK 2.5. Let $\left\{\mathbf{e}_{1}, \ldots, \mathbf{e}_{m}\right\}$ be the standard basis of $\mathfrak{t}^{m}$ whose dual basis are $\left\{\partial_{1}, \ldots, \partial_{m}\right\}$. If $\rho_{*}\left(\mathbf{e}_{i}\right)=0$, then we can easily show that $K$ and $T^{m}$ can be decomposed into $K=K^{\prime} \times S_{i}$ and $T^{m}=T^{m-1} \times S_{i}$ by using (2.3) and (2.4), where $K^{\prime} \subset T^{m-1}$ and $S_{i}$ is the $i^{\text {th }}$ coordinate circle of $T^{m}$. Then, the hyperKähler moment map $\mu_{H K}$ in (2.6) decomposes into $\mu_{H K}^{\prime}$ and $\mu_{i}$, where $\mu_{H K}^{\prime}$ is the hyperKähler moment map of the $K^{\prime}$-action on $\mathbb{H}^{m-1}$ and $\mu_{i}$ is that of the $S_{i}$-action on $\mathbb{H}$. Because the hyperKähler variety constructed by the $S_{i}$-action on $\mathbb{H}$ is the 0 -dimensional manifold, we may regard that the toric hyperKähler variety in this case is constructed by the hyperKähler quotient of $K^{\prime}$-action on $\mathbb{H}^{m-1}$. Hence, throughout this paper, we assume $\rho_{*}\left(\mathbf{e}_{i}\right) \neq 0$ for all $i=1, \ldots, m$.

\section{Hyperplane arrangements}

One of the most important properties of toric hyperKähler manifolds is the correspondence between toric hyperKähler manifolds and hyperplane arrangements, established by Bielawski-Dancer in $[\mathbf{B i D a}]$ (also see $[\mathbf{H a P r}, \mathbf{K o 3}, \mathbf{P r}]$ ). In this section, we recall the hyperplane arrangement induced from the toric hyperKähler variety and recall some basic facts. 
3.1. Hyperplane arrangements induced from toric hyperKähler varieties. In order to define toric hyperKähler varieties, it is enough to use the exact sequence (2.5) and the non-zero element $\alpha \in \mathfrak{k}^{*}$ (see Section 2). By the exactness of (2.5), there is a non-zero lift $\widehat{\alpha}$ of $\alpha$, i.e., $\iota^{*}(\widehat{\alpha})=\alpha$. This $\widehat{\alpha}$ determines $m$ affine hyperplanes in $\left(\mathfrak{t}^{n}\right)^{*}$ as follows:

$$
H_{i}=\left\{x \in\left(\mathfrak{t}^{n}\right)^{*} \mid\left\langle\rho^{*}(x)+\widehat{\alpha}, \mathbf{e}_{i}\right\rangle=0\right\}
$$

where $\mathbf{e}_{i}$ 's $(i=1, \ldots, m)$ are the basis of $\mathfrak{t}^{m} \simeq \mathbb{R}^{m}$ such as those in Remark 2.5.

Remark 3.1. Due to Remark 2.5, we may assume that $\rho_{*}\left(\mathbf{e}_{i}\right) \neq 0$ for all $i=1, \ldots, m$. This implies that $H_{i}$ defined as above is always codimension-one affine subspace in $\left(\mathfrak{t}^{n}\right)^{*}$, i.e., $\operatorname{dim} H_{i}=n-1$. Moreover, we may regard $H_{i}(i=1, \ldots, m)$ as a weighted, cooriented, affine hyperplane by regarding $\rho_{*}\left(\mathbf{e}_{i}\right) \in \mathfrak{t}_{\mathbb{Z}}^{n}$ as a nonzero integer, cooriented, normal vector of $H_{i}$. Here, "weighted" means that $\rho_{*}\left(\mathbf{e}_{i}\right)$ is not required to be primitive.

We call the set of hyperplanes

$$
\mathcal{H}_{\widehat{\alpha}}=\left\{H_{1}, \ldots, H_{m}\right\}
$$

the hyperplane arrangement induced from $\left(M_{\alpha}, T^{n}\right)$ or hyperplane arrangement of $\left(M_{\alpha}, T^{n}\right)$.

REMARK 3.2. Ths choise of a lift $\widehat{\alpha} \in\left(\iota^{*}\right)^{-1}(\alpha)$ yields a parallel translation of the hyperplane arrangement $\mathcal{H}_{\widehat{\alpha}}$. Let $\widehat{\alpha}_{1}$ and $\widehat{\alpha}_{2}$ be elements in $\left(\mathfrak{t}^{n}\right)^{*}$ which satisfy that $\iota^{*}\left(\widehat{\alpha}_{1}\right)=\alpha=\iota^{*}\left(\widehat{\alpha}_{2}\right)$. It is easy to check that the intersection posets of $\mathcal{H}_{\widehat{\alpha}_{1}}$ and $\mathcal{H}_{\widehat{\alpha}_{2}}$ are the same. Namely, the choice of a lift $\widehat{\alpha}$ does not change the combinatorial structure of $\mathcal{H}_{\widehat{\alpha}}$. However, geometrically, $\widehat{\alpha}$ determines the hyperKähler moment map because of the definition in (2.7). This implies that the date $\left(M_{\alpha}, T^{n}, \mu_{\widehat{\alpha}}\right)$ gives more precise structure of hyperplane arrangements than the combinatorial structure (see Section 4.2, 4.3).

We also note that all hyperplane arrangements do not appear as hyperplane arrangements of $\left(M_{\alpha}, T^{n}\right)$. We shall explain it in the following Lemma 3.3. Before that, we prepare two notations. A hyperplane arrangement is called simple, if every nonempty intersection of $k$ hyperplanes is codimension- $k$ and there are $n$ hyperplanes whose intersection is nonempty (also see Figure 3 in Section 3.4). A hyperplane arrangement is called smooth, if it is simple and every collection of $n$ linearly independent vectors $\left\{\rho_{*}\left(\mathbf{e}_{i_{1}}\right), \ldots, \rho_{*}\left(\mathbf{e}_{i_{n}}\right)\right\}$ spans $\mathfrak{t}_{\mathbb{Z}}^{n}$ (also see Figure 2 in Section 3.4). Let us state Lemma 3.3 (see [BiDa, $\mathbf{H a P r}]$ for detail).

LEMMA 3.3. The toric hyperKähler variety $M$ is an orbifold if and only if a hyperplane arrangement of $(M, T)$ is simple. Furthermore, $M$ is smooth (i.e., non-singular) if and only if the hyperplane arrangement of $(M, T)$ is smooth.

The following example is one of the standard examples.

ExAmple 3.4. As seen in Example 2.4, the toric hyperKähler manifold which is defined by the diagonal subgroup $\Delta$ in $T^{n+1}$ is $T^{n}$-equivariantly diffeomorphic to $T^{*} \mathbb{C} P^{n}$.

By using the exactness of (2.8), we may define a representation $\rho^{*}$ as follows:

$$
\rho^{*}\left(x_{1}, \ldots, x_{n}\right)=\left(x_{1}, \ldots, x_{n},-\left(x_{1}+\cdots+x_{n}\right)\right) \in\left(\mathfrak{t}^{n+1}\right)^{*}
$$

Because we took a lift of $\alpha$ as $(1, \ldots, 1) \in\left(\mathfrak{t}^{n+1}\right)^{*}$ (see Example 2.4), by definition, we get the following hyperplanes:

$$
\begin{aligned}
H_{1} & =\left\{\left(x_{1}, \ldots, x_{n}\right) \in\left(\mathfrak{t}^{n}\right)^{*} \mid x_{1}=-1\right\} ; \\
& \vdots \\
H_{n} & =\left\{\left(x_{1}, \ldots, x_{n}\right) \in\left(\mathfrak{t}^{n}\right)^{*} \mid x_{n}=-1\right\} ; \\
H_{n+1} & =\left\{\left(x_{1}, \ldots, x_{n}\right) \in\left(\mathfrak{t}^{n}\right)^{*} \mid x_{1}+\cdots+x_{n}=1\right\} .
\end{aligned}
$$

Figure 1 shows the case when $n=2$.

Henceforth, we assume all toric hyperKähler varieties are non-singular, i.e., all toric hyperKähler varieties are smooth manifolds. In other words, all toric hyperKähler varieties satisfy the condition in Proposition 2.1 or equivalently their hyperplane arrangements are smooth as we have seen in Lemma 3.3. 


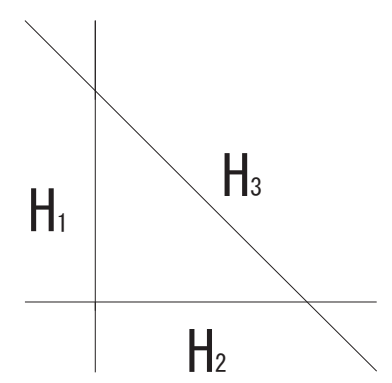

Figure 1. The hyperplane arrangement $\mathcal{H}_{(1,1,1)}$ of $T^{*} \mathbb{C} P(2)$

3.2. Toric hyperKähler manifolds induced from smooth hyperplane arrangements. We next demonstrate the construction of the toric hyperKähler manifold with the hyperKähler moment map $\left(M_{\alpha}^{4 n}, T^{n}, \mu_{\widehat{\alpha}}\right)$ from the smooth hyperplane arrangement in $\left(\mathfrak{t}^{n}\right)^{*}$. Before demonstrating that, we assume the following fact.

REMARK 3.5. If $m=n$, i.e., $\operatorname{dim} \mathbb{H}^{m}=4 \operatorname{dim} T^{n}$, then the toric hyperKähler manifold is $\mathbb{H}^{n}$ itself and the hyperKähler moment map is given by $\mu_{\mathbb{R}} \oplus \mu_{\mathbb{C}}$ (see Section 2.1). Therefore, this case is uniquely determined. If $n=0$, then the toric hyperKähler manifold is the one point and the hyperKähler moment map is given by the zero map to $\{0\} \oplus\{0\}$. Therefore, this case is also uniquely determined. Hence, in this paper, we assume $m \geq n+1$ and $n \geq 1$.

Let $\mathcal{H}$ be a set of weighted, cooriented, affine hyperplanes $\left\{H_{1}, \ldots, H_{m}\right\}$ such that

$$
H_{i}=\left\{x \in\left(\mathfrak{t}^{n}\right)^{*} \mid\left\langle x, v_{i}\right\rangle+\alpha_{i}=0\right\}
$$

and

$$
\operatorname{dim} H_{i}=n-1,
$$

where $v_{i} \in \mathfrak{t}_{\mathbb{Z}}^{n}(i=1, \ldots, m)$ regarded as the weighted coorientation (normal vector) of $H_{i}$ and $\alpha_{i} \in \mathbb{R}$ determines the position in $\left(\mathfrak{t}^{n}\right)^{*}$. Assume the hyperplane arrangement $\mathcal{H}$ is smooth (see Lemma 3.3). Because $\mathcal{H}$ is smooth and $m \geq n+1$ (see Remark 3.5), the surjective homomorphism $\rho_{*}: \mathfrak{t}^{m} \rightarrow \mathfrak{t}^{n}$ can be defined by

$$
\rho_{*}\left(\mathbf{e}_{i}\right)=v_{i} \in \mathfrak{t}_{\mathbb{Z}}^{n},
$$

where $\mathbf{e}_{i}$ is the $i^{\text {th }}$ standard basis of $\mathfrak{t}^{m} \simeq \mathbb{R}^{m}$. Because $\operatorname{dim} H_{i}=n-1$, we have that $v_{i} \neq 0$ (also see Remark 2.5). Put $\mathfrak{k}=\operatorname{ker} \rho_{*}$. Then, there is the following exact sequence:

$$
\{0\} \longrightarrow \mathfrak{k}=\operatorname{ker} \rho_{*} \stackrel{\iota_{*}}{\longrightarrow} \mathfrak{t}^{m} \stackrel{\rho_{*}}{\longrightarrow} \mathfrak{t}^{n} \longrightarrow\{0\} .
$$

Taking its dual, we can define the following sequence as well as the sequence (2.5):

$$
\{0\} \longrightarrow\left(\mathfrak{t}^{n}\right)^{*} \stackrel{\rho^{*}}{\longrightarrow}\left(\mathfrak{t}^{m}\right)^{*} \stackrel{\iota^{*}}{\longrightarrow} \mathfrak{k}^{*} \longrightarrow\{0\} .
$$

Now we may regard $\alpha_{i}=\left\langle\widehat{\alpha}, \mathbf{e}_{i}\right\rangle$ by taking $\widehat{\alpha}=\left(\alpha_{1}, \ldots, \alpha_{m}\right) \in\left(\mathfrak{t}^{m}\right)^{*}$ with respect to the dual basis $\partial_{i}$ of $\mathbf{e}_{i}$. Because $\mathcal{H}$ is simple and $m \geq n+1$, we can easily show that $\iota^{*}(\widehat{\alpha})=\alpha \neq 0$ in $\mathfrak{k}^{*}$. Therefore, with the method similar to that demonstrated in Section 2.1, we can construct the toric hyperKähler variety $M_{\alpha}=\mu_{H K}^{-1}(\alpha, 0) / K$ from the above exact sequence and the nonzero element $\alpha \in \mathfrak{k}^{*}$, where $K$ is the connected torus whose Lie algebra is $\mathfrak{k}$. Moreover, it is easy to check that $M_{\alpha}$ is non-singular by the smoothness of $\mathcal{H}$ and Proposition 2.1, and we have the hyperKähler moment map $\mu_{\widehat{\alpha}}$ as the equation (2.7). Therefore, from the smooth, weighted, cooriented, affine hyperplane arrangement, the toric hyperKähler manifold with the hyperKähler moment map $\left(M_{\alpha}, T^{n}, \mu_{\widehat{\alpha}}\right)$ can be constructed. 
3.3. Geometric meaning of weighted, cooriented, affine hyperplanes. In this subsection, we quickly review the geometric meaning of hyperplanes.

According to [BiDa, Theorem $3.1(1)]$, the hyperKähler moment map $\mu_{\widehat{\alpha}}: M_{\alpha} \rightarrow\left(\mathfrak{t}^{n}\right)^{*} \oplus\left(\mathfrak{t}_{\mathbb{C}}^{n}\right)^{*}$ in (2.7) is surjective. Let $r_{\mathbb{R}}:\left(\mathfrak{t}^{n}\right)^{*} \oplus\left(\mathfrak{t}_{\mathbb{C}}^{n}\right)^{*} \rightarrow\left(\mathfrak{t}^{n}\right)^{*}$ be the natural projection to the real part. Then, we have the surjective map

$$
\Psi_{\widehat{\alpha}}: M_{\alpha} \longrightarrow\left(\mathfrak{t}^{n}\right)^{*}
$$

by $\Psi_{\widehat{\alpha}}=r_{\mathbb{R}} \circ \mu_{\widehat{\alpha}}$.

Let $M_{i}$ be a characteristic submanifold of $\left(M_{\alpha}, T^{n}\right)$, i.e., $M_{i}$ is an invariant connected submanifold in $M_{\alpha}$ which is fixed by some circle subgroup in $T^{n}$. By [HaHo, Section 3], we have the following proposition.

Proposition 3.6. Let $N_{i}(i=1, \ldots, m)$ be the subset in $\mu_{H K}^{-1}(\alpha, 0) \subset \mathbb{C}^{m} \oplus \overline{\mathbb{C}^{m}}$ such that $z_{i}=0=w_{i}$, where $z_{i}$ and $w_{i}$ are the $i^{\text {th }}$ coordinate in $\mathbb{C}^{m}$ and $\overline{\mathbb{C}^{m}}$, respectively. For the given characteristic submanifold $M_{i}$, there exists the subset $N_{i}$ such that

$$
M_{i}=N_{i} / K
$$

Equivalently, $M_{i}$ is the hyperKähler quotient of the restricted $K$-action on the $(m-1)$-dimensional subspace $\mathbb{H}_{i}^{m-1}$, where $\mathbb{H}_{i}^{m-1}$ is the subspace of $\mathbb{H}^{m}$ whose $i^{\text {th }}$ coordinate is 0 .

Due to Proposition 3.6, we have $\operatorname{dim} M_{i}=4 n-4$ for all $i=1, \ldots, m$. By the definition of $\mu_{\widehat{\alpha}}$ and hyperplanes $H_{i}$ (for $i=1, \ldots, m$ ), we have the following relation:

$$
\Psi_{\widehat{\alpha}}\left(M_{i}\right)=H_{i} \subset\left(\mathfrak{t}^{n}\right)^{*} .
$$

Hence, there exists the one-to-one correspondence between hyperplanes in $\mathcal{H}_{\widehat{\alpha}}$ and characteristic submanifolds in $\left(M_{\alpha}, T^{n}, \mu_{\widehat{\alpha}}\right)$.

Next we demonstrate the meaning of the cooriented, normal vector $\rho_{*}\left(\mathbf{e}_{i}\right)$ of $H_{i}$. Note that the vector $\rho_{*}\left(\mathbf{e}_{i}\right)$ is a primitive vector in $\mathfrak{t}^{n}$, because hyperplane arrangements are smooth. By using Proposition 3.6, the circle subgroup which fixes $M_{i}$ is induced by the $i^{\text {th }}$ coordinate circle $S_{i}$ in $T^{m}$. Because $\rho_{*}\left(\mathbf{e}_{i}\right) \neq 0$ in $\mathfrak{t}^{n}$ (see Remark 2.5), the induced subgroup $S_{i} / K\left(\subset T^{m} / K\right)$ is the circle subgroup $T_{i}$ in $T^{n}$ and can be obtained by the exponent of $\rho_{*}\left(\mathbf{e}_{i}\right) \in \mathfrak{t}^{n}$. In other words, such circle subgroup can be determined by the vector $\rho_{*}\left(\mathbf{e}_{i}\right) \in \mathfrak{t}^{n}$ up to sign. Moreover, it is easy to check that the circle subgroup $T_{i}$ acts on the normal bundle $\nu_{i}$ of $M_{i}$ and this action is induced by the right scaler multiplications on fibres, where fibers are isomorphic to $\mathbb{H}=\mathbb{C} \oplus \overline{\mathbb{C}}$. Namely, two choices of signs of $\rho_{*}\left(\mathbf{e}_{i}\right) \in \mathfrak{t}^{n}$ correspond to two orientations of $\nu_{i}$ (we often call the orientation of $\nu_{i}$ an omni-orientation of $M_{i}$, also see [HaMa]). In summary, we have the following corollary as the geometric meaning of the weighted, cooriented, normal vector $\rho_{*}\left(\mathbf{e}_{i}\right)$ of $H_{i}$.

COROLlary 3.7. Let $\rho_{*}\left(\boldsymbol{e}_{i}\right)$ be the weighted, cooriented, normal vector of $H_{i}$. Then, the circle subgroup which fixes the characteristic submanifold $M_{i}$ such that $\Psi_{\widehat{\alpha}}\left(M_{i}\right)=H_{i}$ is given the following subgroup:

$$
T_{i}=\left\{\exp r \rho_{*}\left(\boldsymbol{e}_{i}\right) \mid r \in \mathbb{R}\right\}
$$

for all $i=1, \ldots, m$.

Furthermore, two signs of $\rho_{*}\left(\boldsymbol{e}_{i}\right)$ correspond to two orientations of the normal bundle $\nu_{i}$ of $M_{i}$

3.4. Some remarks on smooth hyperplane arrangements. In closing this section, we give some remarks about smooth hyperplane arrangements. Let $\mathcal{H}_{\widehat{\alpha}}$ be a smooth weighted, cooriented affine hyperplane arrangement. Then, by definition, we may put

$$
\mathcal{H}_{\widehat{\alpha}}=\left\{H_{1}, \ldots, H_{m}\right\}
$$

and

such that

$$
H_{i}=\left\{x \in\left(\mathfrak{t}^{n}\right)^{*} \mid\left\langle x, \rho_{*}\left(\mathbf{e}_{i}\right)\right\rangle=-\left\langle\widehat{\alpha}, \mathbf{e}_{i}\right\rangle\right\},
$$

$$
\left\langle\rho_{*}\left(\mathbf{e}_{i_{1}}\right), \ldots, \rho_{*}\left(\mathbf{e}_{i_{n}}\right)\right\rangle_{\mathbb{Z}}=\mathfrak{t}_{\mathbb{Z}}^{n}
$$


if $\left\{\rho_{*}\left(\mathbf{e}_{i_{1}}\right), \ldots, \rho_{*}\left(\mathbf{e}_{i_{n}}\right)\right\}$ is linearly independent. This condition is equivalent to the following condition:

$$
\operatorname{det}\left(\rho_{*}\left(\mathbf{e}_{i_{1}}\right) \cdots \rho_{*}\left(\mathbf{e}_{i_{n}}\right)\right)= \pm 1
$$

where $\left(\rho_{*}\left(\mathbf{e}_{i_{1}}\right) \cdots \rho_{*}\left(\mathbf{e}_{i_{n}}\right)\right)$ is the $n \times n$-integer matrix whose column vectors consist of $\rho_{*}\left(\mathbf{e}_{i}\right)$ 's.

Because $\mathcal{H}_{\widehat{\alpha}}$ is also simple, we have that for all $i \in[m]$ there exists $\left\{i_{1}, \ldots, i_{n}\right\} \subset[m]$, where $[m]=\{1, \ldots, m\}$, such that

$$
i \in\left\{i_{1}, \ldots, i_{n}\right\}
$$

and

$$
\cap_{j=1}^{n} H_{i_{j}}=\{p\} \subset\left(\mathfrak{t}^{n}\right)^{*} .
$$

By changing the order of hyperplanes, we may regard $\cap_{i=1}^{n} H_{i}=\{p\}$. Let $\rho_{*}\left(\mathbf{e}_{i}\right)=v_{i} \in \mathfrak{t}^{n}$. Then, there exists the linear isomorphism $f_{*}: \mathfrak{t}^{n} \rightarrow \mathfrak{t}^{n}$ such that

$$
f_{*}\left(v_{i}\right)=(0, \ldots, 0,1,0, \ldots, 0)=\mathbf{x}_{i} \in \mathfrak{t}^{n},
$$

i.e., $\left\{f_{*}\left(v_{1}\right), \ldots, f_{*}\left(v_{n}\right)\right\}$ is the standard basis of $\mathfrak{t}^{n}$. By the definition of $H_{i}$ and $p \in H_{i}$ for all $i=1, \ldots, n$, we have

$$
\left\langle\left(f^{*}\right)^{-1}(p), \mathbf{x}_{i}\right\rangle=\left\langle p, f_{*}^{-1}\left(\mathbf{x}_{i}\right)\right\rangle=\left\langle p, v_{i}\right\rangle=-\left\langle\widehat{\alpha}, \mathbf{e}_{i}\right\rangle
$$

for all $i=1, \ldots, n$, where $f^{*}:\left(\mathfrak{t}^{n}\right)^{*} \rightarrow\left(\mathfrak{t}^{n}\right)^{*}$ is the induced dual isomorphism of $f_{*}$. Therefore, we can easily check that there exists an affine (parallel) isomorphism $\mathbb{A}:\left(\mathfrak{t}^{n}\right)^{*} \rightarrow\left(\mathfrak{t}^{n}\right)^{*}$ such that $\mathbb{A}(p)$ is the origin of $\left(\mathfrak{t}^{n}\right)^{*}$ and

$$
\mathbb{A}\left(H_{i}\right)=\left\{x \in\left(\mathfrak{t}^{n}\right)^{*} \mid\left\langle x, \mathbf{x}_{i}\right\rangle=0\right\}
$$

for $i=1, \ldots, n$. Because $\mathbf{x}_{i}$ is the standard basis of $\mathfrak{t}^{n}$, we may regard $\mathbb{A}\left(H_{i}\right)$ as the following linear subspace in $\left(\mathfrak{t}^{n}\right)^{*}$ :

$$
\left(\mathfrak{t}^{n}\right)_{i}^{*}=\left\{\left(x_{1}, \ldots, x_{i-1}, 0, x_{i+1}, \ldots, x_{n}\right) \in\left(\mathfrak{t}^{n}\right)^{*} \mid x_{j} \in\left(\mathfrak{t}^{1}\right)^{*} \simeq \mathbb{R}\right\} .
$$

Therefore, up to affine isomorphism on $\left(\mathfrak{t}^{n}\right)^{*}$, we may regard first $n$ hyperplanes in $\mathcal{H}_{\widehat{\alpha}}$ as $\left(\mathfrak{t}^{n}\right)_{1}^{*}, \ldots,\left(\mathfrak{t}^{n}\right)_{n}^{*}$.

In this case, by the condition (3.3), if there is the hyperplane $H_{n+1} \in \mathcal{H}_{\widehat{\alpha}}$ such that $H_{n+1}$ is not parallel to any $\left(\mathfrak{t}^{n}\right)_{i}^{*}$ for $i=1, \ldots, n$, then $H_{n+1}$ is given by the following hyperplane:

$$
H_{n+1}=\left\{x \in\left(\mathfrak{t}^{n}\right)^{*} \mid\left\langle x, \rho_{*}\left(\mathbf{e}_{n+1}\right)\right\rangle=\left\langle\widehat{\alpha}, \mathbf{e}_{n+1}\right\rangle\right\}
$$

such that $\left\langle\widehat{\alpha}, \mathbf{e}_{n+1}\right\rangle \neq 0$ and

$$
\rho_{*}\left(\mathbf{e}_{n+1}\right)=( \pm 1, \ldots, \pm 1) \in \mathfrak{t}^{n} .
$$

Again by using (3.3), we have that if $H_{n+1}$ and $H_{n+2}$ are not parallel to any $\left(\mathfrak{t}^{n}\right)_{i}^{*}$ then $H_{n+1}$ and $H_{n+2}$ are parallel, i.e.,

$$
\rho_{*}\left(\mathbf{e}_{n+1}\right)=\rho_{*}\left(\mathbf{e}_{n+2}\right) .
$$

Therefore, if $H \in \mathcal{H}_{\widehat{\alpha}}$ is not parallel to any $\left(\mathfrak{t}^{n}\right)_{i}^{*}$ then we may take

$$
\rho_{*}\left(\mathbf{e}_{n+1}\right)=(1, \ldots, 1) \in \mathfrak{t}^{n}
$$

up to linear isomorphism (just changing the sign of some coordinates, i.e., the coorientations of hyperplanes).

Now, we call two hyperplane arrangements $\mathcal{H}_{1}$ and $\mathcal{H}_{2}$ in $\mathbb{R}^{n}$ are affine equivalent if there exists an affine isomorphism $\mathbb{A}: \mathbb{R}^{n} \rightarrow \mathbb{R}^{n}$ which preserves all hyperplanes, i.e., the cardinalities of $\mathcal{H}_{1}$ and $\mathcal{H}_{2}$ are the same, and for all $H_{i}^{(1)} \in \mathcal{H}_{1}$ there exists $H_{j}^{(2)} \in \mathcal{H}_{2}$ such that $\mathbb{A}\left(H_{i}^{(1)}\right)=H_{j}^{(2)}$. 
By using the arguments above, a smooth hyperplane arrangement $\mathcal{H}_{\widehat{\alpha}}$ can be divided into the following subsets up to affine equivalence:

$$
\begin{aligned}
\mathcal{H}_{\widehat{\alpha}}(1) & =\left\{H_{1,1}, \ldots, H_{1, m_{1}}\right\} ; \\
& \vdots \\
\mathcal{H}_{\widehat{\alpha}}(n) & =\left\{H_{n, 1}, \ldots, H_{n, m_{n}}\right\} ; \\
\mathcal{H}_{\widehat{\alpha}}(n+1) & =\left\{H_{n+1,1}, \ldots, H_{n+1, m_{n+1}}\right\} ;
\end{aligned}
$$

where $\mathcal{H}_{\widehat{\alpha}}(i)$ is the set of hyperplanes which are parallel to $\left(\mathfrak{t}^{n}\right)_{i}^{*}$ for $i=1, \ldots, n$ and $\mathcal{H}_{\widehat{\alpha}}(n+1)$ is the set of hyperplanes whose $\rho_{*}\left(\mathbf{e}_{j}\right)$ coincides with $(1, \ldots, 1) \in \mathfrak{t}^{n}$. Here, $\sum_{i=1}^{n} m_{i}+m_{n+1}=m$, $m_{i}>0$ for $i=1, \ldots, n$, and $m_{n+1} \geq 0$.

Therefore, we have established the following proposition (also see Figures 2 and 3):

Proposition 3.8. Let $\mathcal{H}_{\widehat{\alpha}}$ be a smooth hyperplane arrangement. Then, there exists integers $m_{1}, \ldots, m_{n}(>0)$ and $m_{n+1}(\geq 0)$ such that $\mathcal{H}_{\widehat{\alpha}}$ is combinatorially equivalent to the following hyperplane arrangement:

$$
\mathcal{H}\left(m_{1}, \ldots, m_{n}, m_{n+1}\right),
$$

where $\mathcal{H}\left(m_{1}, \ldots, m_{n}, m_{n+1}\right)$ is a simple hyperplane arrangement such that the cardinality of hyperplanes which is parallel to $\left(\mathfrak{t}^{n}\right)_{i}^{*}$ is $m_{i}$ for $i=1, \ldots, n$, and that of hyperplanes whose $\rho_{*}\left(\boldsymbol{e}_{j}\right)$ coincides with $(1, \ldots, 1)$ is $m_{n+1}$.

Furthermore, the above equivalence can be taken from the affine equivalence, for any fixed affine structure on $\mathcal{H}\left(m_{1}, \ldots, m_{n}, m_{n+1}\right)$.
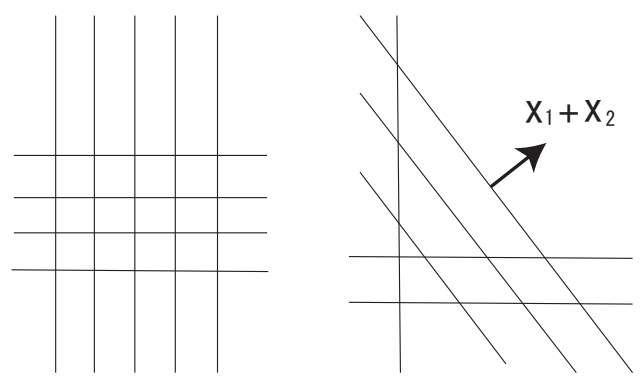

Figure 2. Smooth hyperplane arrangements. The left arrangement is the arrangement represented by $\mathcal{H}(4,5,0)$ and the right one is $\mathcal{H}(2,1,3)$.
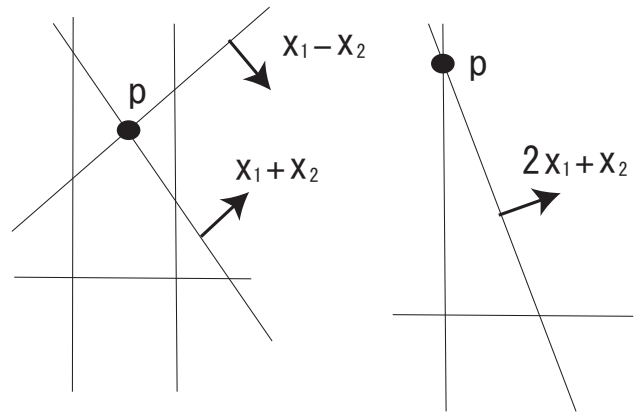

FiguRE 3. Non-smooth hyperplane arrangements. Both of the hyperplanes do not satisfy the condition (3.3) around the vertex $p$; therefore, both of them are not smooth (but simple). Note that bounded regions of smooth hyperplane arrangements in $\mathbb{R}^{2}$ are equilateral triangles or quadrangle only. 
Here, in Proposition 3.8, we call two hyperplanes $\mathcal{H}_{1}$ and $\mathcal{H}_{2}$ are combinatorially equivalent if their intersection posets are equivalent.

\section{Equivalence relations on toric hyperKähler manifolds}

We next define the equivalence relations on toric hyperKähler manifolds and the hyperplane arrangements, and prove that these equivalence relations are compatible (see Theorem 4.4) in this section.

Before we define equivalence relations, we recall the following notations. For two $G$-spaces $X$ and $Y$, a map $f: X \rightarrow Y$ is called a weak $G$-equivariant map if there is a group isomorphism $\varphi: G \rightarrow G$ such that $f(x \cdot t)=f(x) \cdot \varphi(t)$ for all $t \in G$ and $x \in X$; if this isomorphism $\varphi$ is the identity map then $f$ is called a $G$-equivariant map.

4.1. Equivalence relations of toric hyperKähler manifolds. Let $\left(M_{\alpha}, T^{n}, \mu_{\widehat{\alpha}}\right)$ and $\left(M_{\beta}, T^{n}, \mu_{\widehat{\beta}}\right)$ be two toric hyperKähler manifolds with hyperKähler moment maps, where we put $\alpha \in \mathfrak{k}_{1}^{*}, \beta \in \mathfrak{k}_{2}^{*}$ and their lifts $\widehat{\alpha} \in \mathfrak{t}_{1}^{*}, \widehat{\beta} \in \mathfrak{t}_{2}^{*}$, respectively. Here, $\operatorname{dim} T_{1}-\operatorname{dim} K_{1}=n=$ $\operatorname{dim} T_{2}-\operatorname{dim} K_{2}\left(\operatorname{dim} T_{1}=m_{1}\right.$ and $\left.\operatorname{dim} T_{2}=m_{2}\right)$.

DEFINITION 4.1. We say a weakly $T^{n}$-equivariantly isometric map $f: M_{\alpha} \rightarrow M_{\beta}$ a weak hyperhamiltonian $T^{n}$-isometry or weak $T^{n}$-isomorphism simply, if a weak $T^{n}$-equivariant diffeomorphism $f$ preserves the hyperKähler structures and satisfies that

$$
\mu_{\widehat{\alpha}}=\varphi_{\mathbb{R} \oplus \mathbb{C}}^{*} \circ \mu_{\widehat{\beta}} \circ f
$$

that is, the following diagram is commute:

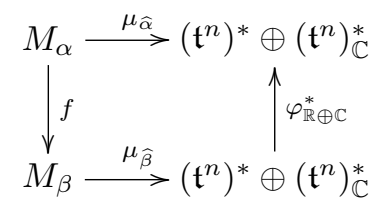

where $\varphi_{\mathbb{R} \oplus \mathbb{C}}^{*}:\left(\mathfrak{t}^{n}\right)^{*} \oplus\left(\mathfrak{t}_{\mathbb{C}}^{n}\right)^{*} \rightarrow\left(\mathfrak{t}^{n}\right)^{*} \oplus\left(\mathfrak{t}_{\mathbb{C}}^{n}\right)^{*}$ is the induced isomorphism from $\varphi: T^{n} \rightarrow T^{n}$ such that $f(x \cdot t)=f(x) \cdot \varphi(t)$. If $\varphi$ is the identity map, then $f$ is called a hyperhamiltonian $T^{n}$-isometry or $T^{n}$-isomorphism simply.

If there is a (weak) $T^{n}$-isomorphism between two triples

$$
\left(M_{\alpha}, T^{n}, \mu_{\widehat{\alpha}}\right) \text { and }\left(M_{\beta}, T^{n}, \mu_{\widehat{\beta}}\right),
$$

then we say that such triples are (weakly) hyperhamiltonian $T^{n}$-isometric or (weakly) $T^{n}$-isomorphic.

REMARK 4.2. In the paper $[\mathbf{B i}]$, if the above $\varphi$ is identity then $M_{\alpha}$ and $M_{\beta}$ are called isomorphic as tri-Hamiltonian hyperKähler T-manifolds.

In this paper, the symbol $\left(M_{\alpha}, T^{n}, \mu_{\widehat{\alpha}}\right) \equiv_{w}\left(M_{\beta}, T^{n}, \mu_{\widehat{\beta}}\right)\left(\operatorname{resp} .\left(M_{\alpha}, T^{n}, \mu_{\widehat{\alpha}}\right) \equiv\left(M_{\beta}, T^{n}, \mu_{\widehat{\beta}}\right)\right)$ represents that $\left(M_{\alpha}, T^{n}, \mu_{\widehat{\alpha}}\right)$ and $\left(M_{\beta}, T^{n}, \mu_{\widehat{\beta}}\right)$ are weakly $T^{n}$-isomorphic (resp. $T^{n}$-isomorphic).

4.2. Equivalence relations of hyperplane arrangements. In this subsection, we introduce the equivalence relations of weighted, cooriented, smooth hyperplane arrangements. Let $\mathcal{H}_{\widehat{\alpha}}=\left\{H_{1}^{(1)}, \ldots, H_{m_{1}}^{(1)}\right\}$ and $\mathcal{H}_{\widehat{\beta}}=\left\{H_{1}^{(2)}, \ldots, H_{m_{2}}^{(2)}\right\}$ be such hyperplane arrangements consist of

$$
\begin{aligned}
& H_{i}^{(1)}=\left\{x \in\left(\mathfrak{t}^{n}\right)^{*} \mid\left\langle x, v_{i}^{(1)}\right\rangle+\widehat{\alpha}_{i}=0\right\}, \\
& H_{j}^{(2)}=\left\{x \in\left(\mathfrak{t}^{n}\right)^{*} \mid\left\langle x, v_{j}^{(2)}\right\rangle+\widehat{\beta}_{j}=0\right\},
\end{aligned}
$$

where $v_{i}^{(1)} \in \mathfrak{t}^{n}\left(i=1, \ldots, m_{1}\right)$ and $v_{j}^{(2)} \in \mathfrak{t}^{n}\left(j=1, \ldots, m_{2}\right)$ are weighted, cooriented vectors, $\widehat{\alpha}_{i}, \widehat{\beta}_{j} \in \mathbb{R}$ represent positions of hyperplanes, respectively

Now we may introduce the equivalence relation on the weighted, cooriented, smooth hyperplane arrangements. Two hyperplane arrangements $\mathcal{H}_{\widehat{\alpha}}$ and $\mathcal{H}_{\widehat{\beta}}$ are called weakly (linear) equivalent if there exists a linear isomorphism $\varphi^{*}:\left(\mathfrak{t}^{n}\right)^{*} \rightarrow\left(\mathfrak{t}^{n}\right)^{*}$, induced from an isomorphism 
$\varphi: T^{n} \rightarrow T^{n}$, such that $\varphi^{*}$ sends $\mathcal{H}_{\widehat{\alpha}}$ to $\mathcal{H}_{\widehat{\beta}}$, i.e., $m_{1}=m_{2}=m$ and there is a permutation $\sigma:[m] \rightarrow[m]$ such that

$$
\varphi^{*}\left(H_{i}^{(1)}\right)=H_{\sigma(i)}^{(2)}
$$

for all $i \in[m]$; in this paper, the symbol $[m]$ for some $m \in \mathbb{N}$ represents the finite set $\{1, \ldots, m\}$, and we denote such hyperplane arrangements by $\mathcal{H}_{\widehat{\alpha}} \equiv_{w} \mathcal{H}_{\widehat{\beta}}$. Moreover, if we can take such $\varphi^{*}$ as the identity map, then $\mathcal{H}_{\widehat{\alpha}}$ and $\mathcal{H}_{\widehat{\beta}}$ are said to be equivalent, and we denote them by $\mathcal{H}_{\widehat{\alpha}} \equiv \mathcal{H}_{\widehat{\beta}}$.

REMARK 4.3. As we mentioned in Section 3.4, there are other equivalence relations of hyperplane arrangements, i.e., affine equivalence and combinatorially equivalence. One can easily show that there are the following hierarchy for these equivalence relations:

\begin{tabular}{|c|c|c|c|c|}
\hline weak equivalence & $\subset$ & affine equivalence & $\subset$ & combinatorially equivalence \\
\hline
\end{tabular}

4.3. Relations between equivalent toric hyperKähler manifolds and their hyperplane arrangements. The goal of this subsection is to prove the following theorem:

THEOREM 4.4. Let $\left(M_{\alpha}, T^{n}, \mu_{\widehat{\alpha}}\right),\left(M_{\beta}, T^{n}, \mu_{\widehat{\beta}}\right)$ be two toric hyperKähler manifolds, and $\mathcal{H}_{\widehat{\alpha}}$, $\mathcal{H}_{\widehat{\beta}}$ be their hyperplane arrangements, respectively. Then, the following two statements are equivalent:

(1) $\left(M_{\alpha}, T^{n}, \mu_{\widehat{\alpha}}\right) \equiv_{w}\left(M_{\beta}, T^{n}, \mu_{\widehat{\beta}}\right)$;

(2) $\mathcal{H}_{\widehat{\alpha}} \equiv{ }_{w} \mathcal{H}_{\widehat{\beta}}$.

We first show the direction $(1) \Rightarrow(2)$ in Theorem 4.4 .

Proposition 4.5. Under the hypothesis of Theorem 4.4, if $\left(M_{\alpha}, T^{n}, \mu_{\widehat{\alpha}}\right) \equiv_{w}\left(M_{\beta}, T^{n}, \mu_{\widehat{\beta}}\right)$, then $\mathcal{H}_{\widehat{\alpha}} \equiv_{w} \mathcal{H}_{\widehat{\beta}}$.

Proof. Assume $\left(M_{\alpha}, T^{n}, \mu_{\widehat{\alpha}}\right) \equiv_{w}\left(M_{\beta}, T^{n}, \mu_{\widehat{\beta}}\right)$. By definition, there exists a weak $T^{n_{-}}$ isomorphism $f: M_{\alpha} \rightarrow M_{\beta}$ such that the following diagram commutes (see Section 3.3 and 4.1):

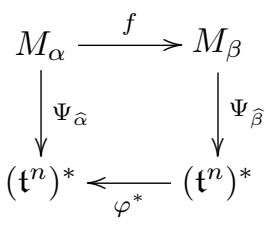

where $\varphi^{*}$ is induced from the isomorphism $\varphi: T^{n} \rightarrow T^{n}$ such that $f(x \cdot t)=f(x) \cdot \varphi(t)$. Because $f$ preserves the characteristic submanifolds, we have that the cardinality of characteristic submanifolds of $M_{\alpha}$ is the same with that of $M_{\beta}$, say $m$. Then, we can define the permutation $\sigma:[m] \rightarrow[m]$ induced by $f$, i.e., if $f\left(M_{i}^{(1)}\right)=M_{j}^{(2)}$ for characteristic submanifolds $M_{i}^{(1)} \subset M_{\alpha}$ and $M_{j}^{(2)} \subset M_{\beta}(i, j \in[m])$, then we define $\sigma(i)=j$. Using the geometric meaning of hyperplanes mentioned in Section 3.3, we have that

$$
\begin{aligned}
H_{i}^{(1)} & =\Psi_{\widehat{\alpha}}\left(M_{i}^{(1)}\right) & & \text { (by the relation }(3.2)) \\
& =\varphi^{*} \circ \Psi_{\widehat{\beta}} \circ f\left(M_{i}^{(1)}\right) & & \text { (by the commutativity of the above diagram) } \\
& =\varphi^{*} \circ \Psi_{\widehat{\beta}}\left(M_{\sigma(i)}^{(2)}\right) & & \text { (by the definition of } \sigma) \\
& =\varphi^{*}\left(H_{\sigma(i)}^{(2)}\right) & & \text { (by the relation }(3.2)),
\end{aligned}
$$

for all $i \in[m]$. This implies that $\varphi^{*}:\left(\mathfrak{t}^{n}\right)^{*} \rightarrow\left(\mathfrak{t}^{n}\right)^{*}$ is a linear map which gives $\mathcal{H}_{\widehat{\alpha}} \equiv_{w} \mathcal{H}_{\widehat{\beta}}$.

The following lemma is the key lemma to prove Theorem 4.4:

LEMmA 4.6. Let $\mathcal{H}_{\widehat{\alpha}}$ and $\mathcal{H}_{\widehat{\beta}}$ be smooth hyperplane arrangements induced from toric hyperKähler manifolds $\left(M_{\alpha}, T^{n}, \mu_{\widehat{\alpha}}\right)$ and $\left(M_{\beta}, T^{n}, \mu_{\widehat{\beta}}\right)$, respectively. Assume a linear isomorphism 
$\varphi^{*}:\left(\mathfrak{t}^{n}\right)^{*} \rightarrow\left(\mathfrak{t}^{n}\right)^{*}$, induced from an isomorphism $\varphi: T^{n} \rightarrow T^{n}$, gives $\mathcal{H}_{\widehat{\beta}} \equiv_{w} \mathcal{H}_{\widehat{\alpha}}$. Then, there exists the lift $\widetilde{\varphi}^{*}$ such that the following diagram commutes:

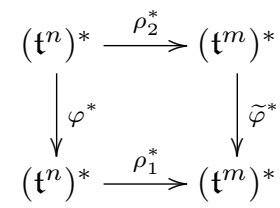

and

$$
\widetilde{\varphi}^{*}(\widehat{\beta})=\widehat{\alpha},
$$

where $\rho_{1}^{*}, \rho_{2}^{*}:\left(\mathfrak{t}^{n}\right)^{*} \rightarrow\left(\mathfrak{t}^{m}\right)^{*}$ are the injective representations defined in (2.5) for $M_{\alpha}, M_{\beta}$, respectively.

Furthermore, the above $\widetilde{\varphi}^{*}$ can be represented as a following matrix:

$$
\left(\begin{array}{cccc}
\epsilon_{1} & 0 & \cdots & 0 \\
0 & \epsilon_{2} & \cdots & 0 \\
\vdots & \vdots & \ddots & \vdots \\
0 & 0 & \cdots & \epsilon_{m}
\end{array}\right) \cdot \Sigma
$$

with respect to the basis $\partial_{i}(i \in[m])$ of $\left(\mathfrak{t}^{m}\right)^{*}$, where $\epsilon_{i}= \pm 1$ for $i \in[m]$ and $\Sigma$ is the $m \times m$ matrix induced from the permutation $\sigma:[m] \rightarrow[m]$.

Proof. Assume $\mathcal{H}_{\widehat{\beta}} \equiv_{w} \mathcal{H}_{\widehat{\alpha}}$. Then, by definition, there exists a linear isomorphism $\varphi^{*}$ : $\left(\mathfrak{t}^{n}\right)^{*} \rightarrow\left(\mathfrak{t}^{n}\right)^{*}$ such that

$$
\varphi^{*}\left(H_{j}^{(2)}\right)=H_{\sigma(j)}^{(1)},
$$

for all $j \in[m]$ and some permutation $\sigma:[m] \rightarrow[m]$, where $H_{\sigma(j)}^{(1)}$ and $H_{j}^{(2)}$ are hyperplanes in $\mathcal{H}_{\widehat{\alpha}}$ and $\mathcal{H}_{\widehat{\beta}}$, respectively. Here, $m$ is the cardinalities of $\mathcal{H}_{\widehat{\alpha}}$ and $\mathcal{H}_{\widehat{\beta}}$. Then, we have the following relations:

$$
\begin{aligned}
& \varphi^{*}\left(H_{j}^{(2)}\right)=\varphi^{*}\left(\left\{x \in\left(\mathfrak{t}^{n}\right)^{*} \mid\left\langle\rho_{2}^{*}(x)+\widehat{\beta}, \mathbf{e}_{j}\right\rangle=0\right\}\right) \\
= & H_{\sigma(j)}^{(1)}=\left\{y \in\left(\mathfrak{t}^{n}\right)^{*} \mid\left\langle\rho_{1}^{*}(y)+\widehat{\alpha}, \mathbf{e}_{\sigma(j)}\right\rangle=0\right\} .
\end{aligned}
$$

Hence, for $x \in H_{j}^{(2)}$, we have the following equations:

$$
\begin{aligned}
& \left\langle\rho_{2}^{*}(x), \mathbf{e}_{j}\right\rangle=-\left\langle\widehat{\beta}, \mathbf{e}_{j}\right\rangle ; \\
& \left\langle\rho_{1}^{*} \circ \varphi^{*}(x), \mathbf{e}_{\sigma(j)}\right\rangle=-\left\langle\widehat{\alpha}, \mathbf{e}_{\sigma(j)}\right\rangle .
\end{aligned}
$$

One can easily show that the following two statements are equivalent:

- $\left\langle\widehat{\beta}, \mathbf{e}_{j}\right\rangle=0$;

- $H_{j}^{(2)}$ is a linear subspace in $\left(\mathfrak{t}^{n}\right)^{*}$.

This implies that $\left\langle\widehat{\beta}, \mathbf{e}_{j}\right\rangle=0$ if and only if $\left\langle\widehat{\alpha}, \mathbf{e}_{\sigma(j)}\right\rangle=0$. Therefore, because $\left\langle\widehat{\alpha}, \mathbf{e}_{\sigma(j)}\right\rangle$ and $\left\langle\widehat{\beta}, \mathbf{e}_{j}\right\rangle$ are real numbers, there exists $\epsilon_{j}^{\prime} \in \mathbb{R} \backslash\{0\}$ such that

$$
\left\langle\widehat{\alpha}, \mathbf{e}_{\sigma(j)}\right\rangle=\epsilon_{j}^{\prime}\left\langle\widehat{\beta}, \mathbf{e}_{j}\right\rangle .
$$

Using the equations (4.4) and (4.5) above, it is easy to check that, for all $x \in H_{j}^{(2)}$,

$$
\left\langle x,\left(\rho_{2}\right)_{*}\left(\epsilon_{j}^{\prime} \mathbf{e}_{j}\right)\right\rangle=\left\langle x, \varphi_{*} \circ\left(\rho_{1}\right)_{*}\left(\mathbf{e}_{\sigma(j)}\right)\right\rangle,
$$

where $\varphi_{*}: \mathfrak{t}^{n} \rightarrow \mathfrak{t}^{n}$ is the dual linear isomorphism of $\varphi^{*}$. Therefore, by using the fact that $H_{j}^{(2)}$ is a codimension one hyperplane, we can easily show that two non-zero vectors $\left(\rho_{2}\right)_{*}\left(\epsilon_{j}^{\prime} \mathbf{e}_{j}\right)$ and $\varphi_{*} \circ\left(\rho_{1}\right)_{*}\left(\mathbf{e}_{\sigma(j)}\right)$ lie in the same 1-dimensional linear subspace in $\mathfrak{t}^{n}$. Hence, there exists $\epsilon_{j} \in \mathbb{R} \backslash\{0\}$ such that

$$
\epsilon_{j}\left(\rho_{2}\right)_{*}\left(\mathbf{e}_{j}\right)=\varphi_{*} \circ\left(\rho_{1}\right)_{*}\left(\mathbf{e}_{\sigma(j)}\right) .
$$


Define the linear map $\widetilde{\varphi}_{*}: \mathfrak{t}^{m} \rightarrow \mathfrak{t}^{m}$ as the following $(m \times m)$-square matrix with respect to the standard basis $\left\{\mathbf{e}_{1}, \ldots, \mathbf{e}_{m}\right\}$ :

$$
\left(\begin{array}{cccc}
\epsilon_{1} & 0 & \cdots & 0 \\
0 & \epsilon_{2} & \cdots & 0 \\
\vdots & \vdots & \ddots & \vdots \\
0 & 0 & \cdots & \epsilon_{m}
\end{array}\right) \cdot \Sigma^{-1}
$$

where the $(m \times m)$-matrix $\Sigma^{-1}$ is the inverse of the matrix $\Sigma$ induced from the permutation $\sigma:[m] \rightarrow[m]$. Using (4.6), we have that the linear map $\widetilde{\varphi}_{*}$ satisfies that

$$
\varphi_{*} \circ\left(\rho_{1}\right)_{*}=\left(\rho_{2}\right)_{*} \circ \widetilde{\varphi}_{*},
$$

i.e., the following diagram is commute:

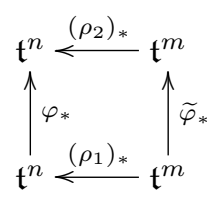

We claim $\epsilon_{j}= \pm 1$ for all $j=1, \ldots, m$. Because $\mathcal{H}_{\widehat{\beta}}$ is a smooth hyperplane arrangements, for all $j \in[m]$, there exists $I_{j} \subset[m]$ such that $\left|I_{j}\right|=n$ (i.e., the cardinality of $I_{j}$ is $n$ ), $j \in I_{j}$, $\cap_{i \in I_{j}} H_{i}^{(2)} \neq \emptyset$ and

$$
\left\{\left(\rho_{2}\right)_{*}\left(\mathbf{e}_{i}\right) \mid i \in I_{j}\right\}
$$

spans $\mathfrak{t}_{\mathbb{Z}}^{n}$ (also see Section 3.4). Therefore, because $\varphi^{*}$ gives weak equivalence between two smooth hyperplane arrangements $\mathcal{H}_{\widehat{\beta}}$ and $\mathcal{H}_{\widehat{\alpha}}$, the vectors

$$
\left\{\left(\rho_{1}\right)_{*}\left(\mathbf{e}_{\sigma(i)}\right) \mid i \in I_{j}\right\}
$$

also spans $\mathfrak{t}_{\mathbb{Z}}^{n}$. By the definition of $\widetilde{\varphi}_{*}$ and the commutativity of the diagram above, we have that

$$
\begin{aligned}
\varphi_{*} \circ\left(\rho_{1}\right)_{*}\left(\mathbf{e}_{\sigma(i)}\right) & =\left(\rho_{2}\right)_{*} \circ \widetilde{\varphi}_{*}\left(\mathbf{e}_{\sigma(i)}\right) \\
& =\left(\rho_{2}\right)_{*}\left(\epsilon_{i} \mathbf{e}_{i}\right) .
\end{aligned}
$$

Because the isomorphism $\varphi_{*}: \mathfrak{t}^{n} \rightarrow \mathfrak{t}^{n}$ is induced from an isomorphism $\varphi: T^{n} \rightarrow T^{n}$, the restriction of $\varphi_{*}$ to $\mathfrak{t}_{\mathbb{Z}}^{n}$ induces the isomorphism between $\mathfrak{t}_{\mathbb{Z}}^{n}$ and $\mathfrak{t}_{\mathbb{Z}}^{n}$. This implies that $\epsilon_{i}= \pm 1$ for all $i \in I_{j}$. Because this satisfies for all $I_{j}(j \in[m])$, we have $\epsilon_{j}= \pm 1$ for all $j=1, \ldots, m$. By taking the dual of the above homomorphisms, it is easy to check the statement of Lemma 4.6.

Now we may prove the direction $(2) \Rightarrow(1)$ in Theorem 4.4 .

Proposition 4.7. Under the hypothesis of Theorem 4.4, if $\mathcal{H}_{\widehat{\alpha}} \equiv_{w} \mathcal{H}_{\widehat{\beta}}$, then $\left(M_{\alpha}, T^{n}, \mu_{\widehat{\alpha}}\right) \equiv_{w}$ $\left(M_{\beta}, T^{n}, \mu_{\widehat{\beta}}\right)$.

Proof. Assume $\mathcal{H}_{\widehat{\alpha}} \equiv_{w} \mathcal{H}_{\widehat{\beta}}$ and this is given by $\varphi^{*}:\left(\mathfrak{t}^{n}\right)^{*} \rightarrow\left(\mathfrak{t}^{n}\right)^{*}$. In this case, by using the arguments in Section 3.3, the cardinality of characteristic submanifolds of $M_{\alpha}$ and that of $M_{\beta}$ are the same, say $m$. Therefore, it also follows from Proposition 3.6 that $M_{\alpha}$ and $M_{\beta}$ are defined by the hyperKähler quotient of torus actions on $\mathbb{H}^{m}$, i.e., there exist the same dimensional subtori $K_{1}, K_{2} \subset T^{m}$ such that

$$
\begin{aligned}
M_{\alpha} & =\mu_{H K}^{-1}(\alpha, 0) / K_{1}, \\
M_{\beta} & =\mu_{H K}^{-1}(\beta, 0) / K_{2},
\end{aligned}
$$

where $K_{s}, s=1,2$, can be defined by the exponent of Lie algebra $\mathfrak{k}_{s}$ whose dual is $\mathfrak{k}_{s}^{*}=\left(\mathfrak{t}^{m}\right)^{*} / \operatorname{Im} \rho_{s}^{*}$. By using Lemma 4.6, there exists the lift $\widetilde{\varphi}^{*}:\left(\mathfrak{t}^{m}\right)^{*} \rightarrow\left(\mathfrak{t}^{m}\right)^{*}$ of $\varphi^{*}$ such that

$$
\widetilde{\varphi}^{*}(\widehat{\beta})=\widehat{\alpha},
$$

and we also have its matrix representation with respect to the basis $\partial_{i}(i \in[m])$ of $\left(\mathfrak{t}^{m}\right)^{*}$ such as the matrix in Lemma 4.6. We denote this matrix representation by $X \in O(\mathrm{~m})$. 
Let $\mu=\mu_{\mathbb{R}} \oplus \mu_{\mathbb{C}}$ be the hyperKähler moment map of the standard $T^{m}$-action on $\mathbb{H}^{m}$ (see Section 2.1). Then, by the definition of $\mu$, it is easy to check that the following diagram is commutative:

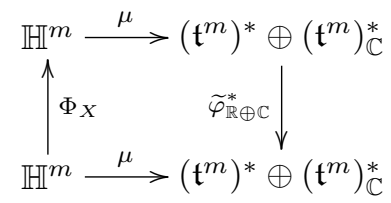

where $\widetilde{\varphi}_{\mathbb{R} \oplus \mathbb{C}}^{*}=\widetilde{\varphi}^{*} \oplus \widetilde{\varphi}_{\mathbb{C}}^{*}(=X \oplus X)$ and $\Phi_{X}: \mathbb{H}^{m} \rightarrow \mathbb{H}^{m}$ is defined by the following matrix $X_{\mathbb{H}}$ in $S p(m)$ :

$$
\left(\begin{array}{cccc}
h_{1} & 0 & \cdots & 0 \\
0 & h_{2} & \cdots & 0 \\
\vdots & \vdots & \ddots & \vdots \\
0 & 0 & \cdots & h_{m}
\end{array}\right) \cdot \Sigma_{\mathbb{H}}^{-1}
$$

such that

$$
\begin{cases}h_{i}=1 & \text { if } \epsilon_{i}=1 \\ h_{i}=\mathbf{k} & \text { if } \epsilon_{i}=-1\end{cases}
$$

and $\Sigma_{\mathbb{H}}^{-1}$ is the $(m \times m)$-matrix induced by the permutation $\sigma^{-1}:[m] \rightarrow[m]$ acting on $\mathbb{H}^{m}$. Here, $\mathbf{k}$ is the basis of $\mathbb{H}$, i.e., $\{1, \mathbf{i}, \mathbf{j}, \mathbf{k}\}$, and $X_{\mathbb{H}}$ acts on $\mathbb{H}^{m}$ from the right hand side (note that $X$ acts on $\left(\mathfrak{t}^{m}\right)^{*} \oplus\left(\mathfrak{t}^{m}\right)_{\mathbb{C}}^{*}$ from the left hand side). Because $\Phi_{X}: \mathbb{H}^{m} \rightarrow \mathbb{H}^{m}$ is represented by $X_{\mathbb{H}} \in S p(m)$ as above and $r \mathbf{k}=\mathbf{k} r^{-1}$ for all $r \in S^{1}$, we see that $\Phi_{X}$ gives the weak $T^{m}$ isomorphism on $\left(\mathbb{H}^{m}, T^{m}, \mu\right)$ such that $\Phi_{X}((z, w) t)=\Phi_{X}(z, w) \widetilde{\varphi}(t)$, where $\widetilde{\varphi}: T^{m} \rightarrow T^{m}$ is the induced isomorphism from $\widetilde{\varphi}^{*}:\left(\mathfrak{t}^{m}\right)^{*} \rightarrow\left(\mathfrak{t}^{m}\right)^{*}$ (also see $\widetilde{\varphi}_{*}$ in the proof of Lemma 4.6).

Because $\widetilde{\varphi}^{*}(\widehat{\beta})=\widehat{\alpha}$, one can easily show that

$$
\Phi_{X}\left(\mu_{H K}^{-1}(\alpha, 0)\right)=\mu_{H K}^{-1}(\beta, 0) .
$$

Recall that, in the first paragraph of this proof, we define $K_{s}, s=1,2$, as the induced torus from $\mathfrak{k}_{s}^{*}=\left(\mathfrak{t}^{m}\right)^{*} / \operatorname{Im} \rho_{s}^{*}$. Because $\widetilde{\varphi}^{*}$ is a lift of $\varphi^{*}$, i.e., $\widetilde{\varphi}^{*} \circ \rho_{2}^{*}=\rho_{1}^{*} \circ \varphi^{*}$, this lift $\widetilde{\varphi}^{*}$ induces the natural isomorphism between $\mathfrak{k}_{2}^{*}$ and $\mathfrak{k}_{1}^{*}$. This implies that

$$
\widetilde{\varphi}\left(K_{1}\right)=K_{2}
$$

Therefore, $\Phi_{X}$ induces the weak $T^{n}$-equivariant diffeomorphism between $M_{\alpha}=\mu_{H K}^{-1}(\alpha, 0) / K_{1}$ and $M_{\beta}=\mu_{H K}^{-1}(\beta, 0) / K_{2}$; moreover, $\Phi_{X}$ preserves their hyperKähler structures because $X_{\mathbb{H}} \in$ $S p(m)$. Note that $\widetilde{\varphi}: T^{m} \rightarrow T^{m}$ induces $\widetilde{\varphi} / K: T^{m} / K_{1} \rightarrow T^{m} / K_{2}$ and $\widetilde{\varphi} / K$ coincides with $\varphi: T^{n} \rightarrow T^{n}$, where $\varphi$ is the isomorphism which induces $\varphi^{*}:\left(\mathfrak{t}^{n}\right)^{*} \rightarrow\left(\mathfrak{t}^{n}\right)^{*}$ in the first paragraph (also see the definition of $\mathcal{H}_{\beta} \equiv_{w} \mathcal{H}_{\alpha}$ in Section 4.2). Moreover, by using $\widetilde{\varphi}^{*}(\widehat{\beta})=\widehat{\alpha}$ and the definitions of the hyperKähler moment maps $\mu_{\widehat{\alpha}}$ and $\mu_{\widehat{\beta}}$ (see Section 2.1.4), we have that the induced diffeomorphism from $\Phi_{X}$ also preserves hyperKähler moment maps $\mu_{\widehat{\alpha}}$ and $\mu_{\widehat{\beta}}$. This establishes that $\left(M_{\alpha}, T^{n}, \mu_{\widehat{\alpha}}\right) \equiv_{w}\left(M_{\beta}, T^{n}, \mu_{\widehat{\beta}}\right)$.

By Propositions 4.5 and 4.7, we have Theorem 4.4.

\section{Equivariant cohomology of toric hyperKähler manifolds}

Using the combinatorial data of the smooth hyperplane arrangement induced from the toric hyperKähler manifold $(M, T)$, we can describe the ring structure of the equivariant cohomology $H_{T}^{*}(M)$ of $(M, T)$, i.e., the Konno's theorem (see Theorem 5.4). In this section, we recall this important fact of the equivariant cohomology of toric hyperKähler manifolds. 
5.1. Notations and $H_{T}^{*}(M)$ as the $H^{*}(B T)$-algebra. We first recall the $H^{*}(B T)$-algebra structure of $H_{T}^{*}(M)$. In order to define $H_{T}^{*}(M)$, we need to take a space $E T \times_{T} M$ called the Borel construction (or homotopy quotient). This space is the orbit space of the diagonal $T$-action on $E T \times M$, where $E T$ is a total space of the universal principal $T$-bundle. Because $T$ acts freely on the $E T$-factor in $E T \times M$, the Borel construction is regarded as the fibre bundle over the classifying space $B T=E T / T$ with fibre $M$, i.e., there is the following fibration:

$$
M \stackrel{j}{\longrightarrow} E T \times_{T} M \stackrel{\pi}{\longrightarrow} B T,
$$

where $\pi$ is the projection and $j$ is the injection to the fixed fibre. We call the ordinary cohomology $H^{*}\left(E T \times_{T} M\right)$ the equivariant cohomology of $(M, T)$, and denote it by $H_{T}^{*}(M)$. By using the fibration (5.1), we have the following induced homomorphism:

$$
\pi^{*}: H^{*}(B T) \longrightarrow H_{T}^{*}(M) .
$$

Hence, the equivariant cohomology $H_{T}^{*}(M)$ can be regarded as not only the ring but also the $H^{*}(B T)$-algebra via $\pi^{*}$.

REMARK 5.1. In this paper, as we mentioned in Section 1, if we do not mention the coefficient of the cohomology, it means that we take the integer $\mathbb{Z}$ as the coefficient ring.

The following proposition is well-known (see e.g. [MiTo, Chapter 3]).

Proposition 5.2. If $\operatorname{dim} T=n$, the cohomology ring $H^{*}(B T ; \mathcal{R})$ is isomorphic to the polynomial ring, i.e.,

$$
H^{*}(B T ; \mathcal{R}) \simeq \mathcal{R}\left[x_{1}, \ldots, x_{n}\right]
$$

for $\mathcal{R}=\mathbb{Z}$ or $\mathbb{R}$, where $\operatorname{deg} x_{i}=2(i=1, \ldots, n)$.

By using the Serre spectral sequence and Proposition 5.2, we also have the following wellknown proposition (see e.g. [MiTo, Chapter 3]).

Proposition 5.3. If $H^{\text {odd }}(M ; \mathcal{R})=0$ and $M$ is simply connected, then the induced homomorphism $\pi^{*}$ is injective and the induced homomorphism $j^{*}: H_{T}^{*}(M ; \mathcal{R}) \rightarrow H^{*}(M ; \mathcal{R})$ is surjective for $\mathcal{R}=\mathbb{Z}$ or $\mathbb{R}$.

In closing this subsection, we recall the equivalence relations on equivariant cohomologies. Let $(M, T)$ and $(N, T)$ be two manifolds with $T$-actions. If there exists ring isomorphisms $f_{T}^{*}$ : $H_{T}^{*}(M) \rightarrow H_{T}^{*}(N)$ and $\varphi^{*}: H^{*}(B T) \rightarrow H^{*}(B T)$ such that the following diagram commutes:

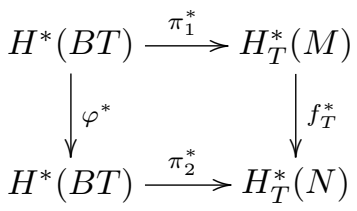

then we call $H_{T}^{*}(M)$ and $H_{T}^{*}(N)$ are weakly $H^{*}(B T)$-algebra isomorphic, and denote them by $H_{T}^{*}(M) \simeq_{w} H_{T}^{*}(N)$. If the above $\varphi^{*}$ is the identity, then we call $H_{T}^{*}(M)$ and $H_{T}^{*}(N)$ are $H^{*}(B T)$ algebra isomorphic, and denote them by $H_{T}^{*}(M) \simeq H_{T}^{*}(N)$.

5.2. Equivariant cohomology of toric hyperKähler manifolds. In this subsection, we review the ring structure of $H_{T}^{*}\left(M_{\alpha}\right)$ of toric hyperKähler manifold $\left(M_{\alpha}, T\right)$ (see e.g. [Ko3, HaHo, HaPr, Pr] for detail).

First, we introduce the ring generators of $H_{T}^{*}\left(M_{\alpha}\right)$ which are defined by the $1^{\text {st }}$ Chern classes of line bundles along the characteristic submanifolds. Let $M_{i}, i=1, \ldots, m$, be the characteristic submanifold of $\left(M_{\alpha}, T\right)$ (see Section 3.3). The symbol $\nu_{i}$ represents its normal bundle in $M_{\alpha}$. Then we may regard the total space $E\left(\nu_{i}\right)$ of $\nu_{i}$ as follows:

$$
E\left(\nu_{i}\right)=N_{i} \times_{K} \mathbb{H}_{i},
$$


by using Proposition 3.6. Here, the 1-dimensional $\mathbb{H}$-vector space $\mathbb{H}_{i}\left(\simeq \mathbb{H} \simeq \mathbb{R}^{4}\right)$ is the representation space of $K$ by the following representation:

$$
\iota_{i}: K \stackrel{\iota}{\longrightarrow} T^{m} \stackrel{p_{i}}{\longrightarrow} S_{i},
$$

where $p_{i}$ is the projection to the $i^{\text {th }}$ coordinate subgroup $S_{i} \simeq S^{1}$ of $T^{m}$. Thus, we may regard the normal bundle $\nu_{i}$ as the pull-back bundle induced from the following diagram:

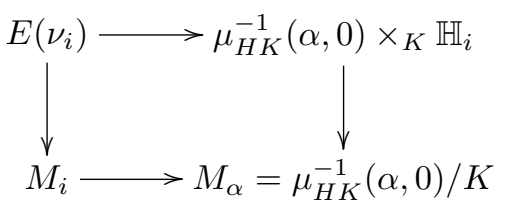

where the bottom $M_{i} \rightarrow M_{\alpha}$ is the inclusion.

Now we may regard the product manifold $\mu_{H K}^{-1}(\alpha, 0) \times \mathbb{H}_{i}$ as the $T^{m}$-manifold, i.e., $T^{m}$ acts on the $\mu_{H K}^{-1}(\alpha, 0)$-factor naturally and on the $\mathbb{H}_{i}$-factor by the representation $p_{i}$ (see (5.3)). Then, the subgroup $K\left(\subset T^{m}\right)$ acts on $\mu_{H K}^{-1}(\alpha, 0) \times \mathbb{H}_{i}$ freely, because $K$ acts on the $\mu_{H K}^{-1}(\alpha, 0)$-factor freely. Therefore, the induced $T^{n}=T^{m} / K$-action on $\mu_{H K}^{-1}(\alpha, 0) \times_{K} \mathbb{H}_{i}$ is well-defined. Similarly, we can define $T^{n}$-action on $E\left(\nu_{i}\right)$. Thus, by taking the Borel construction of each factor in $(5.4)$, the following commutative diagram is induced:

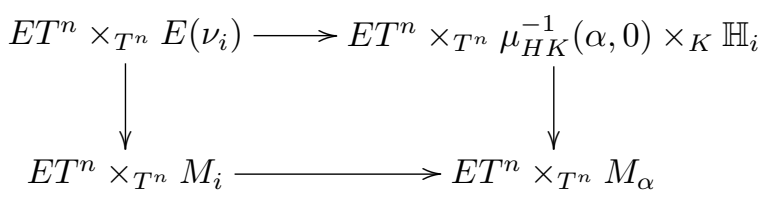

Because $\mathbb{H}=\mathbb{C} \oplus \overline{\mathbb{C}}$ (see Section 2.1), the bundle in (5.5) splits into the following line bundles:

$$
E T^{n} \times_{T^{n}}\left(\mu_{H K}^{-1}(\alpha, 0) \times_{K} \mathbb{H}_{i}\right) \equiv E T^{n} \times_{T^{n}}\left(\mu_{H K}^{-1}(\alpha, 0) \times_{K}\left(\mathbb{C}_{i} \oplus \overline{\mathbb{C}_{i}}\right)\right),
$$

where $\mathbb{C}_{i}$ is the complex 1 -dimensional representation space with $K$-representation via $\iota_{i}$ defined in (5.3), and $\overline{\mathbb{C}}_{i}$ is isomorphic to $\mathbb{C}_{i}$ with reversed orientation. Let $\mathbb{L}_{i}$ be the following line bundle over $E T^{n} \times_{T^{n}} M_{\alpha}$ :

$$
E\left(\mathbb{L}_{i}\right)=E T^{n} \times_{T^{n}}\left(\mu_{H K}^{-1}(\alpha, 0) \times_{K} \mathbb{C}_{i}\right),
$$

and let $\tau_{i}$ be the $1^{\text {st }}$ Chern class of $\mathbb{L}_{i}$, i.e.,

$$
\tau_{i}=c_{1}\left(\mathbb{L}_{i}\right) \in H_{T}^{2}\left(M_{\alpha}\right) .
$$

The following Konno's theorem says that the set of such $1^{\text {st }}$ Chern classes $\left\{\tau_{1}, \ldots, \tau_{m}\right\}$ gives the canonical generator of $H_{T}^{*}\left(M_{\alpha}\right)$ :

Theorem 5.4 (Konno). Let $(M, T)$ be a toric hyperKähler manifold and $\mathcal{H}=\left\{H_{1}, \ldots, H_{m}\right\}$ its hyperplane arrangement. Then, the equivariant cohomology $H_{T}^{*}(M)$ satisfies the following isomorphism:

$$
H_{T}^{*}(M ; \mathbb{Z}) \simeq \mathbb{Z}\left[\tau_{1}, \ldots, \tau_{m}\right] / \mathcal{I}
$$

where $\mathcal{I}$ is the ideal in the polynomial ring $\mathbb{Z}\left[\tau_{1}, \ldots, \tau_{m}\right]$ generated by

$$
\prod_{i \in I} \tau_{i}
$$

for all $I \subset[m]$ such that $\cap_{i \in I} H_{i}=\emptyset$.

Due to the geometric meaning of hyperplanes mentioned in Section 3.3 and the definition of $\tau_{i}$ in (5.7), we have the following correspondence among the characteristic submanifolds $M_{i} \subset M_{\alpha}$, 
the hyperplanes $H_{i} \in \mathcal{H}_{\widehat{\alpha}}$ and the canonical generators $\tau_{i} \in H_{T}^{2}\left(M_{\alpha}\right)$ :

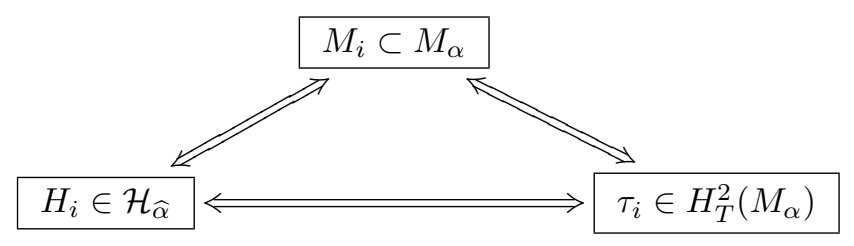

We finish this section by the following example.

ExAmple 5.5. By Example 2.4, the cotangent bundle $T^{*} \mathbb{C} P^{n}$ over $\mathbb{C} P^{n}$ is a toric hyperKähler manifold. Using Example 3.4, we may put the hyperplane arrangement of $T^{*} \mathbb{C} P^{n}$ as $\mathcal{H}=$ $\left\{H_{1}, \ldots, H_{n+1}\right\}$. Again by Example 3.4, if $I \subset[n+1]$ satisfies that $\cap_{i \in I} H_{i}=\emptyset$ then $I=[n+1]$. Therefore, by using Theorem 5.4, the equivariant cohomology ring of $T^{*} \mathbb{C} P^{n}$ is given by

$$
H_{T}^{*}\left(T^{*} \mathbb{C} P^{n}\right) \simeq \mathbb{Z}\left[\tau_{1}, \ldots, \tau_{n+1}\right] /\left\langle\tau_{1} \cdots \tau_{n+1}\right\rangle,
$$

where $\operatorname{deg} \tau_{i}=2$.

In this case, characteristic submanifolds are given by $T^{*} \mathbb{C} P_{i}$, where $\mathbb{C} P_{i} \cong \mathbb{C} P^{n-1}$ is the complex projective space in $\mathbb{C} P^{n}$ whose $i^{\text {th }}$-projective coordinate is 0 .

\section{Hyperplane arrangements induced from the equivariant cohomology}

As we have seen in Section 3 and 4, the hyperplane arrangements induced from toric hyperKähler manifolds are defined in $\left(\mathfrak{t}^{n}\right)^{*}$. One of the idea to prove our main theorem, Theorem 1.1 , is to translate these hyperplane arrangements into the equivariant cohomology $H^{2}(B T ; \mathbb{R})$. In this section, we define the hyperplane arrangement induced from the equivariant cohomology (with fixed generators).

Let $\left(M_{\alpha}, T\right)$ be a toric hyperKähler manifold. Then, by using the homotopy exact sequence for the fibration (5.1), i.e.,

$$
M_{\alpha} \stackrel{j}{\longrightarrow} E T \times_{T} M_{\alpha} \stackrel{\pi}{\longrightarrow} B T,
$$

we have the fundamental group $\pi_{1}\left(E T \times_{T} M_{\alpha}\right)$ is trivial; therefore, $H_{1}\left(E T \times_{T} M_{\alpha}\right)=\{0\}$. Hence, by using the universal coefficient theorem for cohomology, we have

$$
H_{T}^{2}\left(M_{\alpha} ; \mathbb{Z}\right) \simeq \operatorname{Hom}\left(H_{2}\left(E T \times_{T} M_{\alpha}\right), \mathbb{Z}\right) .
$$

Here, the homology $H_{*}\left(E T \times_{T} M_{\alpha}\right)$ is called the equivariant homology, and often denoted by $H_{*}^{T}\left(M_{\alpha}\right)$.

Because of Proposition 5.3 and Theorem 5.4, we have that the following induced exact sequence from the fibration (5.1):

$$
\{0\} \longrightarrow H^{2}(B T ; \mathbb{Z}) \stackrel{\pi^{*}}{\longrightarrow} H_{T}^{2}\left(M_{\alpha} ; \mathbb{Z}\right) \stackrel{j^{*}}{\longrightarrow} H^{2}\left(M_{\alpha} ; \mathbb{Z}\right) \longrightarrow\{0\} .
$$

Now we may take the canonical generator in $H_{T}^{2}\left(M_{\alpha} ; \mathbb{Z}\right)$ as $\left\{\tau_{1}, \ldots, \tau_{m}\right\}$ by virtue of Theorem 5.4. With the method similar to that demonstrated by Masuda in the proof of [Ma2, Proposition 2.2], it is easy to show that the homomorphism $\pi^{*}$ in (6.2) can be expressed as that in the following proposition:

Proposition 6.1. To each $i \in[m]$, there exists a unique element $v_{i} \in H_{2}(B T ; \mathbb{Z})$ such that

$$
\pi^{*}(x)=\sum_{i=1}^{m}\left\langle x, v_{i}\right\rangle_{H} \tau_{i}
$$

for any $x \in H^{2}(B T ; \mathbb{Z})$.

Here, in Proposition 6.1, the symbol $\langle,\rangle_{H}$ represents the pairing of the cohomology and homology defined by $H^{2}(B T) \simeq \operatorname{Hom}\left(H_{2}(B T) ; \mathbb{Z}\right)$ (see $(6.1)$ with $\left.M_{\alpha}=\{*\}\right)$. We also note that Theorem 5.4 and Proposition 6.1 imply that the $H^{*}(B T)$-algebra structure on $H_{T}^{*}\left(M_{\alpha}\right)$. 
Taking the tensor product $H_{T}^{*}\left(M_{\alpha}\right) \otimes_{\mathbb{Z}} \mathbb{R}$, the sequence (6.2) induces the following exact sequence:

$$
\{0\} \longrightarrow H^{2}(B T ; \mathbb{R}) \stackrel{\pi_{\mathbb{R}}^{*}}{\longrightarrow} H_{T}^{2}\left(M_{\alpha} ; \mathbb{R}\right) \stackrel{j_{\mathbb{R}}^{*}}{\longrightarrow} H^{2}\left(M_{\alpha} ; \mathbb{R}\right) \longrightarrow\{0\},
$$

where $\pi_{\mathbb{R}}^{*}$ in $(6.3)$ is induced by $\pi^{*} \otimes i d: H^{*}(B T) \otimes_{\mathbb{Z}} \mathbb{R} \rightarrow H_{T}^{*}\left(M_{\alpha}\right) \otimes_{\mathbb{Z}} \mathbb{R}$. Using Proposition 6.1, the injective homomorphism $\pi_{\mathbb{R}}^{*}$ can be described by the following formula:

$$
\pi_{\mathbb{R}}^{*}(x)=\sum_{i=1}^{m}\left\langle x, v_{i}\right\rangle_{H} \tau_{i},
$$

where $x \in H^{2}(B T ; \mathbb{R}), v_{i} \in H_{2}(B T ; \mathbb{Z}) \subset H_{2}(B T ; \mathbb{R})$ and $\tau_{i} \in H_{T}^{2}\left(M_{\alpha} ; \mathbb{Z}\right) \subset H_{T}^{2}\left(M_{\alpha} ; \mathbb{R}\right)$.

Take an element $\widehat{a} \in H_{T}^{2}\left(M_{\alpha} ; \mathbb{R}\right)$. Then, we define $\mathcal{H}_{\widehat{a}}^{e q}$ as follows:

$$
\mathcal{H}_{\widehat{a}}^{e q}=\left\{H_{1}^{e q}, \ldots, H_{m}^{e q}\right\}
$$

such that each hyperplane $H_{i}^{e q} \subset H^{2}(B T ; \mathbb{R}), i=1, \ldots, m$, is defined by

$$
H_{i}^{e q}=\left\{x \in H^{2}(B T ; \mathbb{R}) \mid\left\langle\pi_{\mathbb{R}}^{*}(x)+\widehat{a}, \mathbf{u}_{i}\right\rangle_{H}=0\right\} .
$$

Here, $\mathbf{u}_{i}, i=1, \ldots, m$, is the linear basis in $H_{2}^{T}(M ; \mathbb{Z})\left(\subset H_{2}^{T}(M ; \mathbb{R})\right)$ such that

$$
\left\langle\tau_{j}, \mathbf{u}_{i}\right\rangle_{H}= \begin{cases}1 & \text { if } i=j \\ 0 & \text { if } i \neq j\end{cases}
$$

where the paring $\langle,\rangle_{H}$ is defined by (6.1). We call $\mathcal{H}_{\widehat{a}}^{e q}$ a hyperplane arrangement of $H_{T}^{*}\left(M_{\alpha}\right)$.

We finish this section by the following two remarks.

REMARK 6.2. The hyperplane arrangement of equivariant cohomology $\mathcal{H}_{\widehat{a}}^{e q}$ is determined by the triple $\left(H_{T}^{2}\left(M_{\alpha}\right), \pi^{*}, \widehat{a}\right)$ for $\widehat{a} \in H_{T}^{2}(M ; \mathbb{R})$ as well as the hyperplane arrangement of toric hyperKähler manifold $\mathcal{H}_{\widehat{\alpha}}$ is determined by the triple $\left(M_{\alpha}, T^{n}, \mu_{\widehat{\alpha}}\right)$ (see Section 2.2 and 3.3). So we may think of the inclusion $\pi^{*}: H^{2}(B T) \rightarrow H_{T}^{2}(M)$ as an algebraic counterpart to the $T$ action on $M_{\alpha}$ and the fixed element $\widehat{a} \in H_{T}^{2}\left(M_{\alpha} ; \mathbb{R}\right)$ as that of the hyperKähler moment map $\mu_{\widehat{\alpha}}: M_{\alpha} \rightarrow\left(\mathfrak{t}^{n}\right)^{*} \oplus\left(\mathfrak{t}^{n}\right)_{\mathbb{C}}^{*}$ (also see [AtBo] and the equivariant symplectic form in [GuSt, Chapter $9])$.

Remark 6.3. Using Proposition 5.3, if $H^{\text {odd }}(M)=0$ then a simply connected $T$-manifold $(M, T)$ (not only toric hyperKähler manifolds) satisfies all conditions mentioned as above. Therefore, for more general class of $T$-manifolds, we can define a hyperplane arrangement of $H_{T}^{*}(M)$ as above.

\section{Equivalence between two hyperplane arrangements in $\left(\mathfrak{t}^{n}\right)^{*}$ and $H^{2}\left(B T^{n} ; \mathbb{R}\right)$}

Henceforth, we assume $\left(M_{\alpha}^{4 n}, T^{n}, \mu_{\widehat{\alpha}}\right)$ is a triple of $4 n$-dimensional toric hyperKähler manifold $M_{\alpha}$, its $T^{n}$-action and its hyperKähler moment map $\mu_{\widehat{\alpha}}$, where $\alpha \in \mathfrak{k}^{*}$ is a non-zero element and $\widehat{\alpha} \in\left(\mathfrak{t}^{m}\right)^{*}$ is its lift (see Section 2). In this section, we prove that two hyperplane arrangements induced from $M_{\alpha}$ and the equivariant cohomology $H_{T}^{*}\left(M_{\alpha}\right)$ are weak equivalent.

7.1. Equivalence of two exact sequences. We first recall the following two exact sequences defined in (2.5) and (6.3):

$$
\{0\} \longrightarrow\left(\mathfrak{t}^{n}\right)^{*} \stackrel{\rho^{*}}{\longrightarrow}\left(\mathfrak{t}^{m}\right)^{*} \stackrel{\iota^{*}}{\longrightarrow} \mathfrak{k}^{*} \longrightarrow\{0\} ;
$$

and

$$
\{0\} \longrightarrow H^{2}(B T ; \mathbb{R}) \stackrel{\pi_{\mathbb{R}}^{*}}{\longrightarrow} H_{T}^{2}\left(M_{\alpha} ; \mathbb{R}\right) \stackrel{j_{\mathbb{R}}^{*}}{\longrightarrow} H^{2}\left(M_{\alpha} ; \mathbb{R}\right) \longrightarrow\{0\} .
$$

In this subsection, we will define the following three natural isomorphisms:

$$
\begin{array}{ll}
J_{n}^{*}:\left(\mathfrak{t}^{n}\right)^{*} \longrightarrow H^{2}\left(B T^{n} ; \mathbb{R}\right) & \text { (see Section 7.1.1); } \\
J_{m}^{*}:\left(\mathfrak{t}^{m}\right)^{*} \longrightarrow H_{T}^{2}\left(M_{\alpha} ; \mathbb{R}\right) & \text { (see Section 7.1.2); } \\
J_{K}^{*}: \mathfrak{k}^{*} \longrightarrow H^{2}\left(M_{\alpha} ; \mathbb{R}\right) & \text { (see Section 7.1.4), }
\end{array}
$$

and prove the following proposition: 
Proposition 7.1. The following diagram (7.3) is a commutative diagram.

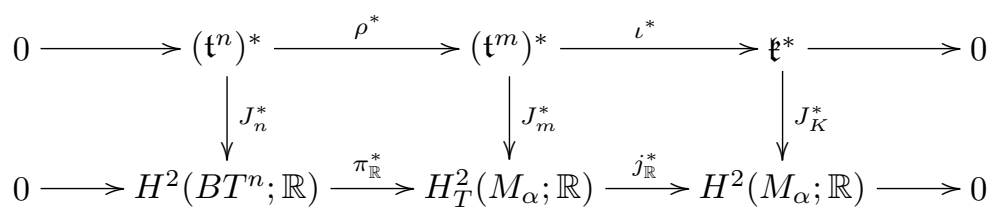

Proposition 7.1 tells us that the two exact sequences (7.1) and (7.2) are equivalent.

7.1.1. Definition of the $1^{\text {st }}$ isomorphism. We first define the $1^{\text {st }}$ isomorphism

$$
J_{n}^{*}:\left(\mathfrak{t}^{n}\right)^{*} \longrightarrow H^{2}\left(B T^{n} ; \mathbb{R}\right) .
$$

Let $f \in \operatorname{Hom}\left(S^{1}, T^{n}\right)$. The homomorphism $f$ can be denoted by

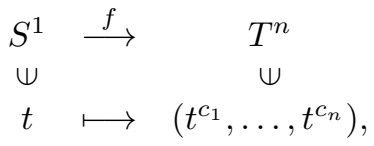

for some integers $c_{1}, \ldots, c_{n}$. Using this (7.5), we have the isomorphism $\operatorname{Hom}\left(S^{1}, T^{n}\right) \simeq \mathbb{Z}^{n}$. Moreover, $f$ induces the continuous map $B f: B S^{1} \rightarrow B T^{n}$, and this map induces the homomorphism $B f_{*}: H_{2}\left(B S^{1} ; \mathbb{Z}\right) \rightarrow H_{2}\left(B T^{n} ; \mathbb{Z}\right)$. We fix a generator $\kappa \in H_{2}\left(B S^{1} ; \mathbb{Z}\right) \simeq \mathbb{Z}$. Because $H_{2}\left(B T^{n} ; \mathbb{Z}\right) \simeq \mathbb{Z}^{n} \simeq \operatorname{Hom}\left(S^{1}, T^{n}\right)$, the homomorphism defined as follow is the isomorphism:

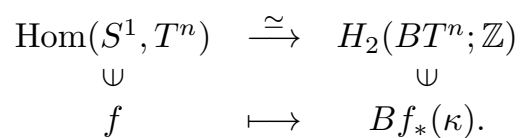

Because of (7.5), there exists the lift to the Lie algebra homomorphism $\widehat{f}: \mathbb{R} \rightarrow \mathfrak{t}^{n}$ such that $\widehat{f}(r)=\left(c_{1} r, \ldots, c_{n} r\right)$ for $r \in \mathbb{R}$, i.e., we have the following commutative diagram

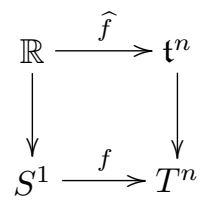

where two vertical maps are the exponential maps from Lie algebras to Lie groups. Because $\left(c_{1}, \ldots, c_{n}\right) \in \mathbb{Z}^{n}$, the lift $\widehat{f}$ preserve lattices, i.e., $\widehat{f}(\mathbb{Z}) \subset \mathfrak{t}_{\mathbb{Z}}^{n}$, where $\mathfrak{t}_{\mathbb{Z}}^{n} \simeq \mathbb{Z}^{n}$ is the lattice in $\mathfrak{t}^{n}$. Hence, there is the following isomorphism:

$$
\begin{array}{cll}
\operatorname{Hom}\left(S^{1}, T^{n}\right) & \stackrel{\simeq}{\longrightarrow} & \mathfrak{t}_{\mathbb{Z}}^{n} \\
\Psi & & \mathbb{\Psi} \\
f & \longmapsto & \widehat{f}(1)=\left(c_{1}, \ldots, c_{n}\right) .
\end{array}
$$

By the composition of two isomorphisms (7.6) and (7.7) as above, we can define the isomorphism

$$
\begin{array}{ccc}
H_{2}\left(B T^{n} ; \mathbb{Z}\right) & \stackrel{\simeq}{\mathfrak{t}_{\mathbb{Z}}^{n}} \\
\Psi & & \Psi \\
B f_{*}(\kappa) & \longmapsto & \widehat{f}(1) .
\end{array}
$$

Taking the tensor products with $\mathbb{R}$, this isomorphism induces the following isomorphism:

$$
\left(J_{n}\right)_{*}: H_{2}\left(B T^{n} ; \mathbb{R}\right) \stackrel{\simeq}{\longrightarrow} \mathfrak{t}^{n} .
$$

The isomorphism (7.4) is define by taking the dual of $\left(J_{n}\right)_{*}$. 
7.1.2. Definition of the $2^{\text {nd }}$ isomorphisms. We next define the $2^{\text {nd }}$ isomorphism

$$
J_{m}^{*}:\left(\mathfrak{t}^{m}\right)^{*} \longrightarrow H_{T}^{2}\left(M_{\alpha} ; \mathbb{R}\right)
$$

as follows:

$$
\begin{array}{ccc}
\left(\mathfrak{t}^{m}\right)^{*} & \stackrel{\simeq}{\longrightarrow} & H_{T}^{2}\left(M_{\alpha} ; \mathbb{R}\right) \\
\Psi & & \Psi \\
\partial_{i} & \stackrel{J_{m}^{*}}{\longmapsto} & \tau_{i},
\end{array}
$$

where $\partial_{i}$ is the dual basis in $\left(\mathfrak{t}^{m}\right)^{*}(i=1, \ldots, m)$ of the basis $\mathbf{e}_{i}$ in $\mathfrak{t}^{m} \simeq \mathbb{R}^{m}$ (see Remark 2.5 ), and $\tau_{i}$ is the canonical generator of the equivariant cohomology $H_{T}^{*}\left(M_{\alpha} ; \mathbb{Z}\right)$ (see $(5.7)$ and Theorem 5.4); here, we may regard it as the basis of $H_{T}^{2}\left(M_{\alpha} ; \mathbb{R}\right)$.

Note that the isomorphism $J_{m}^{*}$ in $(7.9)$ is also defined by the dual of the following isomorphism:

$$
\begin{array}{ccc}
H_{2}^{T}\left(M_{\alpha} ; \mathbb{R}\right) & \stackrel{\left(J_{m}\right)_{*}}{\longrightarrow} & \mathfrak{t}^{m} \\
\Psi & \simeq & \Psi \\
\mathbf{u}_{i} & \longmapsto & \mathbf{e}_{i},
\end{array}
$$

where $\mathbf{u}_{i}$ is the basis which satisfies $(6.5)$.

7.1.3. Preparation to define the $3^{\text {rd }}$ isomorphisms: the geometric interpretation of $v_{i}$. In order to define the $3^{\text {rd }}$ isomorphisms, we recall the geometric meaning of the unique element $v_{i} \in$ $H_{2}(B T ; \mathbb{Z}) \subset H_{2}(B T ; \mathbb{R})$ in the following formulation (6.4) (also see Proposition 6.1);

$$
\pi_{\mathbb{R}}^{*}(u)=\sum_{i=1}^{m}\left\langle u, v_{i}\right\rangle_{H} \tau_{i} \in H_{T}^{2}\left(M_{\alpha} ; \mathbb{R}\right),
$$

for $u \in H^{2}(B T ; \mathbb{R})$. Namely, the purpose of Section 7.1.3 is to prove the following proposition.

Proposition 7.2. Let $T_{i}$ be the circle subgroup in $T^{n}$ appeared in Corollary 3.7, i.e., the circle subgroup which fixes the characteristic submanifold $M_{i}$. Let $\lambda_{v_{i}}: S^{1} \rightarrow T^{n}$ be the homomorphism which corresponds to the unique element $v_{i} \in H_{2}(B T ; \mathbb{Z})$ in Proposition 6.1 via the inverse of isomorphism (7.6). Then, the following relation holds:

$$
\lambda_{v_{i}}\left(S^{1}\right)=T_{i} .
$$

We note that the arguments we will use in Section 7.1.3 is almost similar to the proof of [Ma1, Lemma 1.10].

First, we recall the tangent spaces of fixed points of toric hyperKähler manifolds (see $[\mathbf{H a H o}$, Section 3] for detail). Let $p$ be a fixed point, i.e., $p \in M_{\alpha}^{T}$, and $M_{i} \subset M_{\alpha}$ be the characteristic submanifold (see Section 3.2) $(i=1, \ldots, m)$. Put $I(p)=\left\{i \mid p \in M_{i}^{T}\right\}$. By the definitions of toric hyperKähler manifolds and their characteristic submanifolds (also see Proposition 3.6), we have that the cardinality of $I(p)$ is just $n$ for all $p \in M_{\alpha}^{T}$, i.e., $|I(p)|=n$, and

$$
\{p\}=\cap_{i \in I(p)} M_{i} .
$$

Using (5.2), there exists the following decomposition:

$$
T_{p} M_{\alpha}=\left.\bigoplus_{i \in I(p)} \nu_{i}\right|_{p}=\bigoplus_{i \in I(p)} V\left(\chi_{i}\right) \oplus V\left(-\chi_{i}\right),
$$

where $V\left(\chi_{i}\right) \oplus V\left(-\chi_{i}\right) \simeq \mathbb{C} \oplus \overline{\mathbb{C}} \simeq \mathbb{H}$, the representation $\chi_{i}: T^{n} \rightarrow S^{1}$ is induced from the normal representation of $M_{i}$ on $p$ and $-\chi_{i}$ is its orientation-reversing representation, i.e., $-\chi_{i}(t)=\chi_{i}(t)^{-1}$.

Let us interpret the above $\chi_{i} \in \operatorname{Hom}\left(T^{n}, S^{1}\right)$ as the element in the (equivariant) cohomology $H^{2}\left(B T^{n} ; \mathbb{Z}\right)$ (see Proposition 7.4 ). Taking the dual of (7.6), we first define the following isomorphism:

$$
\begin{array}{ccc}
H^{2}\left(B T^{n} ; \mathbb{Z}\right) & \stackrel{\simeq}{ } & \operatorname{Hom}\left(T^{n}, S^{1}\right) \\
\Psi & & \Psi \\
u & \longmapsto & \chi^{u} .
\end{array}
$$

In order to prove Proposition 7.4, we prepare the following lemma: 
Lemma 7.3. Let $\left(B \chi^{u}\right)^{*}: H^{*}\left(B S^{1} ; \mathbb{Z}\right) \rightarrow H^{*}\left(B T^{n} ; \mathbb{Z}\right)$ be the induced representation. Then, it satisfies that

$$
\left(B \chi^{u}\right)^{*}\left(\kappa^{*}\right)=u
$$

for the dual basis $\kappa^{*} \in H^{2}\left(B S^{1} ; \mathbb{Z}\right)$ of a basis $\kappa \in H_{2}\left(B S^{1} ; \mathbb{Z}\right)$.

Proof. Let $f \in \operatorname{Hom}\left(S^{1}, T^{n}\right)$. Then, by definition, $\chi^{u} \circ f: S^{1} \rightarrow S^{1}$ can be defined by the following homomorphism:

$$
\chi^{u} \circ f(r)=r^{\left\langle u, B f_{*}(\kappa)\right\rangle_{H}},
$$

where $r \in S^{1}$. Because $\kappa^{*}$ is the dual basis, by using the arguments demonstrated in Section 7.1.1 (in this case, $n=1$ ), we have that

$$
\left\langle u, B f_{*}(\kappa)\right\rangle_{H}=\left\langle\kappa^{*},\left(B \chi^{u} \circ f\right)_{*}(\kappa)\right\rangle_{H} .
$$

Because this equation holds for all $f \in \operatorname{Hom}\left(S^{1}, T^{n}\right)$, we have the statement of this lemma.

Let $\iota_{p}$ be the inclusion $\iota_{p}:\{p\} \rightarrow M_{\alpha}$ and $\iota_{p}^{T}$ be its induced inclusion $\iota_{p}^{T}: E T \times_{T}\{p\} \rightarrow$ $E T \times_{T} M_{\alpha}$. The inclusion $\iota_{p}^{T}$ induces the following representation:

$$
\iota_{p}^{*}: H_{T}^{*}\left(M_{\alpha}\right) \rightarrow H_{T}^{*}(\{p\})=H^{*}(B T) .
$$

Then, $\chi_{i}: T^{n} \rightarrow S^{1}$ in (7.10) can be translated into the element in the equivariant cohomology as the following proposition:

Proposition 7.4. For all $i \in I(p), \chi_{i}=\chi^{\iota_{p}^{*}\left(\tau_{i}\right)}$.

Proof. The representation $\chi_{i}: T^{n} \rightarrow S^{1}$ in (7.10) induces the continuous map $B \chi_{i}: B T \rightarrow$ $B S^{1}$. Now we may regard (by changing the sign of $\kappa$ if we need) the basis $\kappa^{*} \in H^{*}\left(B S^{1} ; \mathbb{Z}\right)$ as the $1^{\text {st }}$ Chern class of the canonical line bundle $\eta$ :

$$
E(\eta)=E S^{1} \times_{S^{1}} \mathbb{C} \longrightarrow B S^{1},
$$

where $S^{1}$ acts on $\mathbb{C}$ by the scaler multiplication (rotated by one time only), i.e.,

$$
\kappa^{*}=c_{1}(\eta) .
$$

Let $E T \times_{T} V\left(\chi_{i}\right)$ be the Borel construction of the representation space $V\left(\chi_{i}\right)$. Then we may regard $E T \times_{T} V\left(\chi_{i}\right) \rightarrow B T$ as the line bundle over $B T$ and denote this line bundle as $\gamma_{i}$. It is easy to see that $\gamma_{i}$ is the pull-back of $\eta$ along $B \chi_{i}: B T \rightarrow B S^{1}$, i.e.,

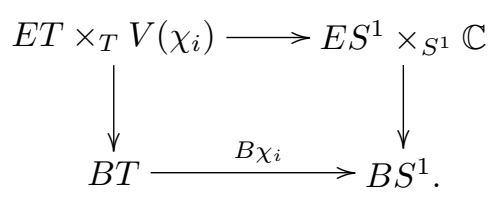

Therefore, $B \chi_{i}^{*}\left(\kappa^{*}\right)=B \chi_{i}^{*} c_{1}(\eta)=c_{1}\left(\gamma_{i}\right)$. Hence, it follows from Lemma 7.3 that $B \chi_{i}^{*}=$ $\left(B \chi^{c_{1}\left(\gamma_{i}\right)}\right)^{*}$. Thus, together with the definition of $J_{n}^{*}$ in Section 7.1.1, we have

$$
\chi_{i}=\chi^{c_{1}\left(\gamma_{i}\right)}
$$

Hence, in order to complete the proof, i.e., to prove $\chi_{i}=\chi^{\iota_{p}^{*}\left(\tau_{i}\right)}$, it is sufficient to show that $c_{1}\left(\gamma_{i}\right)=\iota_{p}^{*}\left(\tau_{i}\right)$

Using (5.5) and (7.10), one can easily show that $\gamma_{i}$ is the pull-back of $\mathbb{L}_{i}$ (see (5.6)) along the following inclusion:

$$
\iota_{p}^{T}: E T \times_{T}\{p\} \hookrightarrow E T \times_{T} M_{i} \hookrightarrow E T \times_{T} M_{\alpha}
$$

Because $c_{1}\left(\mathbb{L}_{i}\right)=\tau_{i}$, we have $c_{1}\left(\gamma_{i}\right)=\iota_{p}^{*}\left(\tau_{i}\right)$. This establishes Proposition 7.4.

Moreover, we have the following corollary.

Corollary 7.5. The set $\left\{\iota_{p}^{*}\left(\tau_{i}\right) \mid i \in I(p)\right\}$ is a basis of $H^{2}(B T ; \mathbb{Z})$ for all $p \in M_{\alpha}^{T}$. 
Proof. It is sufficient to show that $\left\{\chi_{i} \mid i \in I(p)\right\}$ is a basis of $\operatorname{Hom}\left(T^{n}, S^{1}\right)$ by using $(7.11)$ and Lemma 7.4. By the definition of the toric hyperKähler manifold $M_{\alpha}$, we see that $T^{n}$ acts on $M_{\alpha}$ effectively. Hence, by using the differentiable slice theorem (see e.g. $[\mathbf{B r}, \mathbf{K a}]$ ), this action induces an effective and linear $T^{n}$-action on the tangent space $T_{p} M$ for every fixed point $p \in M_{\alpha}^{T}$, in other wards, the induced $T^{n}$-action on $T_{p} M \simeq \mathbb{H}^{n}$ can be defined by an injective representation to $S p(n)$. It follows that the representations $\left\{\chi_{i} \mid i \in I(p)\right\}$ which appeared in the decomposition (7.10) of $T_{p} M$ can be regarded as a basis of $\operatorname{Hom}\left(T^{n}, S^{1}\right)$. This establishes Corollary 7.5.

Using the definition of $\mathbb{L}_{i}$ (see (5.6)), we have that the restricted bundle $\left.\mathbb{L}_{i}\right|_{p}$ to $p \in M^{T} \backslash M_{i}^{T}$ is the trivial line bundle over BT. Therefore, it follows from Corollary 7.5 that we have the following relations:

$$
\begin{cases}\iota_{p}^{*}\left(\tau_{i}\right)=0 & \text { if } p \in M^{T} \backslash M_{i}^{T} \\ \iota_{p}^{*}\left(\tau_{i}\right) \neq 0 & \text { if } p \in M_{i}^{T}\end{cases}
$$

The following lemma tells us the relation between $\iota_{p}^{*}\left(\tau_{i}\right) \in H^{2}(B T)$ and $v_{i} \in H_{2}(B T)$ in Proposition 6.1.

Lemma 7.6. Let $v_{i}$ be the element appearing in Proposition 6.1. Then, the set $\left\{\iota_{p}^{*}\left(\tau_{i}\right) \mid i \in\right.$ $I(p)\} \subset H^{2}(B T ; \mathbb{Z})$ is the dual basis of $\left\{v_{i} \mid i \in I(p)\right\} \subset H_{2}(B T ; \mathbb{Z})$.

Proof. By using Proposition 6.1, we have the following equation:

$$
\pi^{*}\left(\iota_{p}^{*}\left(\tau_{i}\right)\right)=\sum_{j=1}^{m}\left\langle\iota_{p}^{*}\left(\tau_{i}\right), v_{j}\right\rangle_{H} \tau_{j} .
$$

Using (7.12), the equation above implies the following equation:

$$
\iota_{p}^{*} \circ \pi^{*}\left(\iota_{p}^{*}\left(\tau_{i}\right)\right)=\sum_{j \in I(p)}\left\langle\iota_{p}^{*}\left(\tau_{i}\right), v_{j}\right\rangle_{H} \iota_{p}^{*}\left(\tau_{j}\right) .
$$

Because $E T \times_{T}\{p\} \cong B T \stackrel{\iota_{p}}{\longrightarrow} E T \times_{T} M \stackrel{\pi}{\longrightarrow} B T$ can be regarded as the identity map, the induced map $\iota_{p}^{*} \circ \pi^{*}$ is the identity map. Therefore, we have

$$
\iota_{p}^{*}\left(\tau_{i}\right)=\sum_{j \in I(p)}\left\langle\iota_{p}^{*}\left(\tau_{i}\right), v_{j}\right\rangle_{H} \iota_{p}^{*}\left(\tau_{j}\right) .
$$

By Corollary 7.5, there are no linear relations among $\iota_{p}^{*}\left(\tau_{j}\right)$ 's. Therefore we have the following equation:

$$
\begin{cases}\left\langle\iota_{p}^{*}\left(\tau_{i}\right), v_{j}\right\rangle_{H}=0 & \text { if } i \neq j \\ \left\langle\iota_{p}^{*}\left(\tau_{i}\right), v_{j}\right\rangle_{H}=1 & \text { if } i=j .\end{cases}
$$

This establishes that the element $\iota_{p}^{*}\left(\tau_{i}\right)$ is the dual basis of $v_{i}$ for all $i \in I(p)$.

In order to prove Proposition 7.2, we prepare one more lemma:

Lemma 7.7. Let $\chi^{u} \in \operatorname{Hom}\left(T, S^{1}\right)\left(\operatorname{resp} . \lambda_{v} \in \operatorname{Hom}\left(S^{1}, T\right)\right)$ be the corresponding homomorphism to $u \in H^{2}(B T ; \mathbb{Z})$ (resp. $\left.v \in H_{2}(B T ; \mathbb{Z})\right)$ via $(7.11)$ (resp. (7.6)). Then

$$
\chi^{u} \circ \lambda_{v}(r)=r^{\langle u, v\rangle_{H}}
$$

for all $r \in S^{1}$.

Proof. Let $\kappa^{*} \in H^{2}\left(B S^{1} ; \mathbb{Z}\right) \subset H^{2}\left(B S^{1} ; \mathbb{R}\right)$ be the dual basis of $\kappa \in H_{2}\left(B S^{1} ; \mathbb{Z}\right) \subset$ $H_{2}\left(B S^{1} ; \mathbb{R}\right)$. Put $\left(B \chi^{u} \circ B \lambda_{v}\right)_{*}: H_{*}\left(B S^{1} ; \mathbb{R}\right) \rightarrow H_{*}\left(B S^{1} ; \mathbb{R}\right)$ the induced homomorphism by $\chi^{u} \circ \lambda_{v}: S^{1} \rightarrow S^{1}$. Because $\kappa \in H_{2}\left(B S^{1} ; \mathbb{Z}\right)$ is a generator, we can put $\left(B \chi^{u} \circ B \lambda_{v}\right)_{*}(\kappa)=a(u, v) \kappa$ for some $a(u, v) \in \mathbb{Z}$. Using (7.6) and Lemma 7.3, we have

$$
\begin{aligned}
\langle u, v\rangle_{H} & =\left\langle\left(B \chi^{u}\right)^{*}\left(\kappa^{*}\right),\left(B \lambda_{v}\right)_{*}(\kappa)\right\rangle_{H}=\left\langle\kappa^{*}, B \chi_{*}^{u} \circ\left(B \lambda_{v}\right)_{*}(\kappa)\right\rangle_{H} \\
& =\left\langle\kappa^{*},\left(B \chi^{u} \circ B \lambda_{v}\right)_{*}(\kappa)\right\rangle_{H}=\left\langle\kappa^{*}, a(u, v) \kappa\right\rangle_{H} \\
& =a(u, v) .
\end{aligned}
$$


It follows that

$$
\left(B \chi^{u} \circ B \lambda_{v}\right)_{*}(\kappa)=\langle u, v\rangle_{H} \kappa .
$$

Therefore, by using the isomorphism $(7.6),(7.7)$ for $n=1$, we have that

$$
\chi^{u} \circ \lambda_{v}(r)=r^{\langle u, v\rangle_{H}}
$$

for $r \in S^{1}$.

Now we may prove Proposition 7.2.

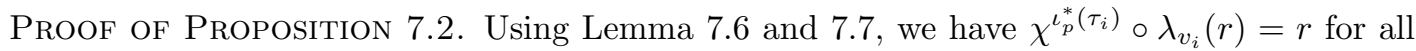
$p \in M_{i}^{T}$ and $r \in S^{1}$. By Proposition 7.4, we also have $\chi_{i} \circ \lambda_{v_{i}}(r)=r$ for all $r \in S^{1}$. It follows from the decomposition (7.10) that $\lambda_{v_{i}}\left(S^{1}\right)$ acts on the normal space of $M_{i}$ on each $p \in M_{i}^{T}$ effectively. Therefore, one can easily show that the circle subgroup $\lambda_{v_{i}}\left(S^{1}\right) \subset T^{n}$ acts trivially on $M_{i}$. Thus, we have that $\lambda_{v_{i}}\left(S^{1}\right)=T_{i}$. This establishes Proposition 7.2.

7.1.4. Definition of the $3^{\text {rd }}$ isomorphisms. We finally define the $3^{\text {rd }}$ isomorphism

$$
J_{K}^{*}: \mathfrak{k}^{*} \longrightarrow H^{2}\left(M_{\alpha} ; \mathbb{R}\right),
$$

and prove Proposition 7.1 .

In order to do that, we first prove the following proposition:

Proposition 7.8. The following diagram is a commutative diagram:

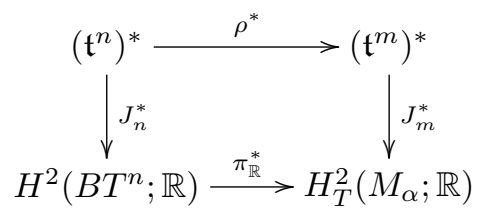

where $\rho^{*}$ is defined by (2.5) (or see $(7.1)$ ), $\pi_{\mathbb{R}}^{*}$ by (6.4), $J_{n}^{*}$ by (7.4) and $J_{m}^{*}$ by (7.9).

In order to prove Proposition 7.8, we prepare the following lemma:

LEMMA 7.9. The following equation holds for all $i=1, \ldots, m$,

$$
\left(J_{n}\right)_{*}\left(v_{i}\right)=\rho_{*}\left(\boldsymbol{e}_{i}\right),
$$

where $v_{i}$ is the unique element appearing in Proposition 6.1.

Proof. Let $\lambda_{v_{i}} \in \operatorname{Hom}\left(S^{1}, T\right)$ be the element which corresponds to $v_{i} \in H_{2}(B T ; \mathbb{Z})$ via the isomorphism (7.6). By the definition of $\left(J_{n}\right)_{*}$ in Section 7.1.1, we have that

$$
\left(J_{n}\right)_{*}\left(v_{i}\right)=\widehat{\lambda}_{v_{i}}(1) \text {, }
$$

where $\widehat{\lambda}_{v_{i}}: \mathbb{R} \rightarrow \mathfrak{t}^{n}$ is the lift of $\lambda_{v_{i}}$. Therefore, it is enough to show that $\widehat{\lambda}_{v_{i}}(1)=\rho_{*}\left(\mathbf{e}_{i}\right)$.

Because $\mathbf{e}_{i} \in \mathfrak{t}_{\mathbb{Z}}^{m}$, we have that $\rho_{*}\left(\mathbf{e}_{i}\right) \in \mathfrak{t}_{\mathbb{Z}}^{n}$. Let $\lambda_{\rho_{*}\left(\mathbf{e}_{i}\right)} \in \operatorname{Hom}\left(S^{1}, T\right)$ be the element which corresponds to $\rho_{*}\left(\mathbf{e}_{i}\right) \in \mathfrak{t}_{\mathbb{Z}}^{n}$ via the isomorphism (7.7), i.e., $\widehat{\lambda}_{\rho_{*}\left(\mathbf{e}_{i}\right)}(1)=\rho_{*}\left(\mathbf{e}_{i}\right)$ for the lift $\widehat{\lambda}_{\rho_{*}\left(\mathbf{e}_{i}\right)}$ : $\mathbb{R} \rightarrow \mathfrak{t}^{n}$. Hence, using Corollary 3.7 and Proposition 7.2 , we have that

$$
\lambda_{\rho_{*}\left(\mathbf{e}_{i}\right)}\left(S^{1}\right)=\left\{\exp r \rho_{*}\left(\mathbf{e}_{i}\right) \mid r \in \mathbb{R}\right\}=T_{i}=\lambda_{v_{i}}\left(S^{1}\right) .
$$

It follows that $\widehat{\lambda}_{\rho_{*}\left(\mathbf{e}_{i}\right)}(1)=\rho_{*}\left(\mathbf{e}_{i}\right)=\widehat{\lambda}_{v_{i}}(1)$. This establishes the statement of Lemma 7.9.

Now we may prove prove Proposition 7.8.

Proof of Proposition 7.8. Let $x \in\left(\mathfrak{t}^{n}\right)^{*}$. Using the formula (6.4), we have the equation

$$
\pi_{\mathbb{R}}^{*} \circ J_{n}^{*}(x)=\sum_{i=1}^{m}\left\langle J_{n}^{*}(x), v_{i}\right\rangle_{H} \tau_{i},
$$

where $v_{i} \in H_{2}(B T ; \mathbb{Z}) \subset H_{2}(B T ; \mathbb{R})$ and $\tau_{i} \in H_{T}^{2}(M ; \mathbb{Z}) \subset H_{T}^{2}(M ; \mathbb{R})(i=1, \ldots, m)$. By the definition of $J_{n}^{*}$ in Section 7.1, we also have the equation

$$
\left\langle J_{n}^{*}(x), v_{i}\right\rangle_{H}=\left\langle x,\left(J_{n}\right)_{*}\left(v_{i}\right)\right\rangle,
$$


where $\left(J_{n}\right)_{*}: H_{2}(B T ; \mathbb{R}) \rightarrow \mathfrak{t}^{n}$ is the isomorphism defined in (7.8). Note that, in the above equation, the left $\langle,\rangle_{H}$ is the pairing of the cohomology and homology and the right $\langle$,$\rangle is the$ pairing of the dual Lie algebra and Lie algebra. Using the above two equations, we have

$$
\pi_{\mathbb{R}}^{*} \circ J_{n}^{*}(x)=\sum_{i=1}^{m}\left\langle x,\left(J_{n}\right)_{*}\left(v_{i}\right)\right\rangle_{H} \tau_{i} .
$$

On the other hand, the homomorphism $\rho^{*}:\left(\mathfrak{t}^{n}\right)^{*} \rightarrow\left(\mathfrak{t}^{m}\right)^{*}$ can be denoted as follows:

$$
\begin{aligned}
\rho^{*}(x) & =\sum_{i=1}^{m}\left\langle\rho^{*}(x), \mathbf{e}_{i}\right\rangle \partial_{i} \\
& =\sum_{i=1}^{m}\left\langle x, \rho_{*}\left(\mathbf{e}_{i}\right)\right\rangle \partial_{i} \in\left(\mathfrak{t}^{m}\right)^{*},
\end{aligned}
$$

where $\mathbf{e}_{i}$ is the natural basis in $\mathfrak{t}^{m}$ and $\partial_{i}$ is its dual basis in $\left(\mathfrak{t}^{m}\right)^{*}$. Therefore, by the definition of $J_{m}^{*}$ in Section 7.1 .2 and $(7.16)$, we have that

$$
J_{m}^{*} \circ \rho^{*}(x)=\sum_{i=1}^{m}\left\langle x, \rho_{*}\left(\mathbf{e}_{i}\right)\right\rangle_{H} \tau_{i}
$$

Using (7.15), (7.17) and Lemma 7.9, we have Proposition 7.8.

Let us define the $3^{\text {rd }}$ isomorphism $J_{K}^{*}$ in (7.13). First, we regard the two homomorphisms in (7.1) and (7.2) as the quotient homomorphism:

$$
\iota^{*}:\left(\mathfrak{t}^{m}\right)^{*} \rightarrow \mathfrak{k}^{*} \simeq\left(\mathfrak{t}^{m}\right)^{*} / \operatorname{Im}\left(\rho^{*}\right)
$$

and

$$
j_{\mathbb{R}}^{*}: H_{T}^{2}(M ; \mathbb{R}) \rightarrow H^{2}(M ; \mathbb{R}) \simeq H_{T}^{2}(M ; \mathbb{R}) / \operatorname{Im}\left(\pi_{\mathbb{R}}^{*}\right) .
$$

Then, using Proposition 7.8, we get the well-defined isomorphism $J_{K}^{*}: \mathfrak{k}^{*} \rightarrow H^{2}(M ; \mathbb{R})$ as the quotient homomorphism of the isomorphism $J_{m}^{*}:\left(\mathfrak{t}^{m}\right)^{*} \rightarrow H_{T}^{2}(M ; \mathbb{R})$ defined by (7.9). This gives the definition of the $3^{\text {rd }}$ isomorphism (7.13).

It is also easy to check Proposition 7.1 by using Proposition 7.8 and the definition of $J_{K}^{*}$.

7.2. Equivalence of two hyperplane arrangements. In this subsection, we prove that the hyperplane arrangement $\mathcal{H}_{\widehat{\alpha}} \subset\left(\mathfrak{t}^{n}\right)^{*}$ defined by $\left(M_{\alpha}, T^{n}, \mu_{\widehat{\alpha}}\right)$ is weak equivalent to the hyperplane arrangement $\mathcal{H}_{\widehat{a}}^{e q} \subset H^{2}(B T ; \mathbb{R})$ defined by the triple $\left(H_{T}^{2}\left(M_{\alpha}\right), \pi^{*}, \widehat{a}\right)$, where $\widehat{a}$ is the element in $H_{T}^{2}\left(M_{\alpha}\right)$ such that $\widehat{a}=J_{m}^{*}(\widehat{\alpha})$. Namely, we prove the isomorphism $J_{n}^{*}:\left(\mathfrak{t}^{n}\right)^{*} \rightarrow H^{2}(B T ; \mathbb{R})$ defined in Section 7.1.1 preserves these two hyperplane arrangements.

We first recall two hyperplane arrangements (see Section 3 and Section 6). Using the top exact sequence in (7.3), the hyperplane arrangement $\mathcal{H}_{\widehat{\alpha}}=\left\{H_{1}, \ldots, H_{m}\right\}$ of $\left(M_{\alpha}, T^{n}, \mu_{\widehat{\alpha}}\right)$ satisfies

$$
H_{i}=\left\{x \in\left(\mathfrak{t}^{n}\right)^{*} \mid\left\langle\rho^{*}(x)+\widehat{\alpha}, \mathbf{e}_{i}\right\rangle=0\right\},
$$

where $\widehat{\alpha} \in\left(\mathfrak{t}^{m}\right)^{*}$ is a lift of the non-zero element $\alpha \in \mathfrak{k}^{*}$ and $\mathbf{e}_{i} \in \mathfrak{t}^{m}$ is the basis whose dual is $\partial_{i} \in\left(\mathfrak{t}^{m}\right)^{*}$. On the other hand, using the bottom exact sequence in (7.3), we have the hyperplane arrangement $\mathcal{H}_{\widehat{a}}^{e q}=\left\{H_{1}^{e q}, \ldots, H_{m}^{e q}\right\}$ of $\left(H_{T}^{*}\left(M_{\alpha}\right), \pi^{*}, \widehat{a}\right)$ for $\widehat{a}\left(=J_{m}^{*}(\widehat{\alpha})\right) \in H_{T}^{2}\left(M_{\alpha} ; \mathbb{R}\right)$ as follows:

$$
H_{i}^{e q}=\left\{y \in H^{2}(B T ; \mathbb{R}) \mid\left\langle\pi_{\mathbb{R}}^{*}(y)+\widehat{a}, \mathbf{u}_{i}\right\rangle=0\right\},
$$

where $\mathbf{u}_{i} \in H_{2}^{T}\left(M_{\alpha} ; \mathbb{R}\right)$ is the basis whose dual is tha canonical generator $\tau_{i} \in H_{T}^{2}(M)$.

In order to prove the weak equivalence of two hyperplane arrangements, we remark the following.

REMARK 7.10. Using the following three facts: $\left(J_{m}\right)_{*}\left(\mathbf{u}_{i}\right)=\mathbf{e}_{i}$ (see Section 7.1 .2$) ; \rho_{*}\left(\mathbf{e}_{i}\right) \neq 0$ (see Remark 3.1); and the commutativity of the dual of the diagram (7.3), we have $\left(\pi_{\mathbb{R}}\right)_{*}\left(\mathbf{u}_{i}\right) \neq 0$ for all $i=1, \ldots, m$. This gives that $\operatorname{dim} H_{i}^{e q}=n-1$.

The following proposition tells us the equivalence of $\mathcal{H}_{\widehat{\alpha}}$ and $\mathcal{H}_{\widehat{a}}^{e q}$. 
Proposition 7.11. Let $\mathcal{H}_{\widehat{\alpha}}=\left\{H_{1}, \ldots, H_{m}\right\}$ be the hyperplane arrangement of $\left(M_{\alpha}, T^{n}, \mu_{\widehat{\alpha}}\right)$ and $\mathcal{H}_{\widehat{a}}^{e q}=\left\{H_{1}^{e q}, \ldots, H_{m}^{e q}\right\}$ that of $\left(H_{T}^{*}\left(M_{\alpha}\right), \pi^{*}, \widehat{a}\right)$, where $\widehat{a}=J_{m}^{*}(\widehat{\alpha})$. Then, the isomorphism $J_{n}^{*}:\left(\mathfrak{t}^{n}\right)^{*} \rightarrow H^{2}(B T ; \mathbb{R})$ satisfies that

$$
J_{n}^{*}\left(H_{i}\right)=H_{i}^{e q}
$$

for all $i=1, \ldots, m$.

In particular, we have that $\mathcal{H}_{\widehat{\alpha}}$ induced from $\left(M_{\alpha}, T^{n}, \mu_{\widehat{\alpha}}\right)$ and $\mathcal{H}_{\widehat{a}}^{\text {eq }}$ induced from $\left(H_{T}^{*}\left(M_{\alpha}\right), \pi^{*}, \widehat{a}\right)$ are weak equivalence (see Section 4.2).

Proof. Let $x \in H_{i} \in \mathcal{H}_{\widehat{\alpha}}$. Then, by definition, $x$ satisfies $\left\langle\rho^{*}(x)+\widehat{\alpha}, \mathbf{e}_{i}\right\rangle=0$. Then, By using the definition of $J_{m}^{*}$ and, we have that $J_{n}^{*}(x) \in H^{2}(B T ; \mathbb{R})$ satisfies the following equations:

$$
\begin{aligned}
& \left\langle\pi_{\mathbb{R}}^{*} \circ J_{n}^{*}(x)+\widehat{a}, \mathbf{u}_{i}\right\rangle_{H} \\
& =\left\langle J_{m}^{*} \circ \rho^{*}(x)+J_{m}^{*}(\widehat{\alpha}), \mathbf{u}_{i}\right\rangle_{H} \quad \text { (by the commutativity of (7.3)) } \\
& =\left\langle\rho^{*}(x)+\widehat{\alpha},\left(J_{m}\right)_{*}\left(\mathbf{u}_{i}\right)\right\rangle \quad \text { (by the definition of } J_{m}^{*} \text { ) } \\
& =\left\langle\rho^{*}(x)+\widehat{\alpha}, \mathbf{e}_{i}\right\rangle \quad\left(\text { by }\left(J_{m}\right)_{*}\left(\mathbf{u}_{i}\right)=\mathbf{e}_{i}\right) \\
& =0 \text {. }
\end{aligned}
$$

Therefore, $J_{n}^{*}\left(H_{i}\right) \subset H_{i}^{e q}$. Because $J_{n}^{*}$ is the isomorphism and $\operatorname{dim} H_{i}=\operatorname{dim} H_{i}^{e q}=n-1$ (by Remark 3.1 and 7.10), we have that

$$
J_{n}^{*}\left(H_{i}\right)=H_{i}^{e q}
$$

for all $i=1, \ldots, m$.

\section{8. $T^{n}$-equivariant cohomological rigidity}

In this final section, we prove the following main theorem of this paper:

TheOREM 8.1. Let $\left(M_{\alpha}, T, \mu_{\widehat{\alpha}}\right)$ and $\left(M_{\beta}, T, \mu_{\widehat{\beta}}\right)$ be toric hyperKähler manifolds with hyperKähler moment maps. The following two statements are equivalent:

(1) $\left(M_{\alpha}, T, \mu_{\widehat{\alpha}}\right) \equiv_{w}\left(M_{\beta}, T, \mu_{\widehat{\beta}}\right)$;

(2) there exists a weak $H^{*}(B T)$-algebra isomorphism $f_{T}^{*}: H_{T}^{*}\left(M_{\alpha} ; \mathbb{Z}\right) \rightarrow H_{T}^{*}\left(M_{\beta} ; \mathbb{Z}\right)$ such that $f_{T}^{*}(\widehat{a})=\widehat{b}$,

where $\widehat{a}=J_{m_{1}}^{*}(\widehat{\alpha})$ and $\widehat{b}=J_{m_{2}}^{*}(\widehat{\beta})$ for the isomorphisms $J_{m_{1}}^{*}:\left(\mathfrak{t}^{m_{1}}\right)^{*} \rightarrow H_{T}^{2}\left(M_{\alpha} ; \mathbb{R}\right)$ and $J_{m_{2}}^{*}$ : $\left(\mathfrak{t}^{m_{2}}\right)^{*} \rightarrow H_{T}^{2}\left(M_{\beta} ; \mathbb{R}\right)$ defined in Section 7.1.2.

Let $\left(M_{\alpha}, T^{n}, \mu_{\widehat{\alpha}}\right),\left(M_{\beta}, T^{n}, \mu_{\widehat{\beta}}\right)$ be two toric hyperKähler manifolds, and $\mathcal{H}_{\widehat{a}}^{e q}$, $\mathcal{H}_{\widehat{b}}^{e q}$ be their hyperplane arrangements induced from the equivariant cohomologies, respectively, where $\widehat{a}=$ $J_{m_{1}}^{*}(\widehat{\alpha})$ and $\widehat{b}=J_{m_{2}}^{*}(\widehat{\beta})$. Here, $M_{\alpha}=\mu_{H K}^{-1}(\alpha, 0) / K_{1}$ such that $\mu_{H K}: \mathbb{H}^{m_{1}} \rightarrow\left(\mathfrak{k}_{1}\right)^{*} \oplus\left(\mathfrak{k}_{1}\right)_{\mathbb{C}}^{*}$ and $M_{\beta}=\mu_{H K}^{-1}(\beta, 0) / K_{2}$ such that $\mu_{H K}: \mathbb{H}^{m_{2}} \rightarrow\left(\mathfrak{k}_{2}\right)^{*} \oplus\left(\mathfrak{k}_{2}\right)_{\mathbb{C}}^{*}$. By using Theorem 4.4 and Proposition 7.11, in order to prove Theorem 8.1, it is enough to show the following two statements are equivalent:

(1) $\mathcal{H}_{\widehat{a}}^{e q} \equiv_{w} \mathcal{H}_{\widehat{b}}^{e q}$ :

(2) there exists a weak $H^{*}(B T)$-algebra isomorphism $f_{T}^{*}: H_{T}^{*}\left(M_{\alpha} ; \mathbb{Z}\right) \rightarrow H_{T}^{*}\left(M_{\beta} ; \mathbb{Z}\right)$ such that $f_{T}^{*}(\widehat{a})=\widehat{b}$.

8.1. Proof of $(1) \Rightarrow(2)$. We first prove the following proposition:

Proposition 8.2. If $\mathcal{H}_{\widehat{a}}^{e q} \equiv_{w} \mathcal{H}_{\widehat{b}}^{e q}$, then there exists a weak $H^{*}(B T)$-algebra isomorphism $f_{T}^{*}: H_{T}^{*}\left(M_{\alpha} ; \mathbb{Z}\right) \rightarrow H_{T}^{*}\left(M_{\beta} ; \mathbb{Z}\right)$ such that $f_{T}^{*}(\widehat{a})=\widehat{b}$.

Proof. Let $\varphi^{*}: H^{2}(B T) \rightarrow H^{2}(B T)$ be an isomorphism such that $\varphi_{\mathbb{R}}^{*}$ gives $\mathcal{H}_{\widehat{a}}^{e q} \equiv_{w} \mathcal{H}_{\widehat{b}}^{e q}$. Then, we may put $m$ as the cardinalities of $\mathcal{H}_{\widehat{a}}^{e q}$ and $\mathcal{H}_{\widehat{b}}^{e q}$. Due to Proposition 7.1, we may regard two exact sequences appearing in Proposition 7.1 as the same sequences. Therefore, with the 
method similar to that demonstrated in the proof of Lemma 4.6, there exists the lift $f_{T}^{*}$ such that the following diagram commutes:

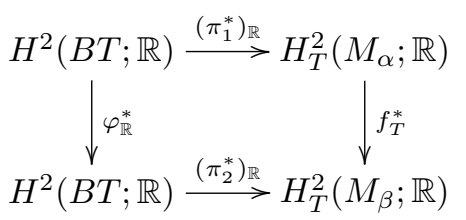

and

$$
f_{T}^{*}(\widehat{a})=\widehat{b}
$$

where $\pi_{1}: E T \times_{T} M_{\alpha} \rightarrow B T$ and $\pi_{2}: E T \times_{T} M_{\beta} \rightarrow B T$ are the projections of the Borel constructions. Furthermore, the above $f_{T}^{*}$ can be represented as the following matrix:

$$
\left(\begin{array}{cccc}
\epsilon_{1} & 0 & \cdots & 0 \\
0 & \epsilon_{2} & \cdots & 0 \\
\vdots & \vdots & \ddots & \vdots \\
0 & 0 & \cdots & \epsilon_{m}
\end{array}\right) \cdot \Sigma
$$

with respect to the canonical basis $\tau_{i}^{(1)}$ of $H_{T}^{2}\left(M_{\alpha}\right)$ and $\tau_{i}^{(2)}$ of $H_{T}^{2}\left(M_{\beta}\right)(i \in[m])$, where $\epsilon_{i}= \pm 1$ and $\Sigma$ is the $(m \times m)$-matrix induced from the permutation $\sigma:[m] \rightarrow[m]$. Namely, for the canonical basis of $H_{T}^{*}\left(M_{\alpha}\right)$ and $H_{T}^{*}\left(M_{\beta}\right)$, we have

$$
f_{T}^{*}\left(\tau_{i}^{(1)}\right)=\epsilon_{i} \tau_{\sigma(i)}^{(2)} .
$$

We claim that this $f_{T}^{*}$ extends to the weak $H^{*}(B T)$-algebra isomorphism. Because $\varphi_{\mathbb{R}}^{*}\left(H_{i}^{(1)}\right)=$ $H_{\sigma(i)}^{(2)}$ for $H_{i}^{(1)} \in \mathcal{H}_{\widehat{a}}^{e q}$ and $H_{\sigma(i)}^{(2)} \in \mathcal{H}_{\widehat{b}}^{e q}$, we have that the following two statements are equivalent for $I \subset[m]$ :

$$
\begin{aligned}
& \text { - } \cap_{i \in I} H_{i}^{(1)}=\emptyset ; \\
& \text { - } \cap_{\sigma(i) \in \sigma(I)} H_{\sigma(i)}^{(2)}=\emptyset .
\end{aligned}
$$

Therefore, due to Theorem 5.4, the linear isomorphism $f_{T}^{*}: H_{T}^{2}\left(M_{\alpha}\right) \rightarrow H_{T}^{2}\left(M_{\beta}\right)$ naturally extends to the ring isomorphism

$$
f_{T}^{*}: H_{T}^{*}\left(M_{\alpha}\right) \longrightarrow H_{T}^{*}\left(M_{\beta}\right) .
$$

Note that $H^{*}(B T)$ is the polynomial ring (see Proposition 5.2), i.e., there is no relations among generators $\left\{x_{1}, \ldots, x_{n}\right\}$ in $H^{*}(B T)$. Therefore, the linear isomorphism $\varphi^{*}: H^{2}(B T) \rightarrow H^{2}(B T)$ also naturally extends to the ring isomorphism

$$
\varphi^{*}: H^{*}(B T) \longrightarrow H^{*}(B T) .
$$

By using Proposition 6.1 and the commutativity of the diagram (8.1), it is easy to check that, for every degree, the following diagram commutes:

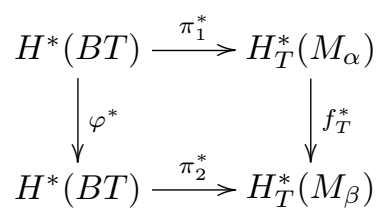

i.e., $f_{T}^{*}$ is a weak $H^{*}(B T)$-algebra isomorphism. 
8.2. Proof of $(2) \Rightarrow(1)$. We next prove the converse of Proposition 8.2. Assume that $f_{T}^{*}: H_{T}^{*}\left(M_{\alpha}\right) \rightarrow H_{T}^{*}\left(M_{\beta}\right)$ is a weak $H^{*}(B T)$-algebra isomorphism such that $f_{T}^{*}(\widehat{a})=\widehat{b}$. Let $\left\{\tau_{1}^{(1)}, \ldots, \tau_{m_{1}}^{(1)}\right\}$ and $\left\{\tau_{1}^{(2)}, \ldots, \tau_{m_{2}}^{(2)}\right\}$ be the canonical generators of $H_{T}^{*}\left(M_{\alpha}\right)$ and $H_{T}^{*}\left(M_{\beta}\right)$, respectively ( say $\mathcal{T}^{(1)}$ and $\mathcal{T}^{(2)}$ briefly).

Because $H_{T}^{*}\left(M_{\alpha}\right) \simeq H_{T}^{*}\left(M_{\beta}\right)$, we have that $m_{1}=m_{2}=m$. The goal of this final subsection is to prove the following proposition:

Proposition 8.3. If there exists a weak $H^{*}(B T)$-algebra isomorphism $f_{T}^{*}: H_{T}^{*}\left(M_{\alpha} ; \mathbb{Z}\right) \rightarrow$ $H_{T}^{*}\left(M_{\beta} ; \mathbb{Z}\right)$ such that $f_{T}^{*}(\widehat{a})=\widehat{b}$, then $\mathcal{H}_{\widehat{a}}^{e q} \equiv{ }_{w} \mathcal{H}_{\widehat{b}}^{e q}$.

In order to prove Proposition 8.3, the most part of this subsection is devoted to the proof of the following key lemma:

LEMmA 8.4. For any weak $H^{*}(B T)$-algebra isomorphism $f_{T}^{*}: H_{T}^{*}\left(M_{\alpha}\right) \rightarrow H_{T}^{*}\left(M_{\beta}\right)$ such that $f_{T}^{*}(\widehat{a})=\widehat{b}$, there exists a weak $H^{*}(B T)$-algebra isomorphism $g_{T}^{*}: H_{T}^{*}\left(M_{\alpha}\right) \rightarrow H_{T}^{*}\left(M_{\beta}\right)$ such that $g_{T}^{*}(\widehat{a})=\widehat{b}$ and $g_{T}^{*}$ preserves the canonical generators up to sign, i.e., there exists a permutation $\sigma:[m] \rightarrow[m]$ and $\epsilon_{i}= \pm 1$ such that

$$
g_{T}^{*}\left(\tau_{i}^{(1)}\right)=\epsilon_{i} \tau_{\sigma(i)}^{(2)}
$$

for all $i \in[m]$.

In order to prove this lemma, we prepare some notations and facts (also see [Ma2]).

Let $M_{\alpha}^{T}$ be the set of $T$-fixed points in $M_{\alpha}$. Because $p \in M_{\alpha}^{T}$ can be represented by

$$
\{p\}=\cap_{j=1}^{n} M_{i_{j}}
$$

for some characteristic submanifolds $M_{i_{j}}\left(j=1, \ldots, n\right.$, where $\left.4 n=\operatorname{dim} M_{\alpha}\right)$, the fixed point set $M_{\alpha}^{T}$ consists of finitely many points.

For $\xi \in H_{T}^{2}\left(M_{\alpha}\right)$, we denote its restriction to $p \in M_{\alpha}^{T}$ by $\left.\xi\right|_{p}$, i,e,

$$
\left.\xi\right|_{p}=\iota_{p}^{*}(\xi) \in H_{T}^{*}(\{p\})=H^{*}(B T),
$$

where $\iota_{p}^{*}$ is the induced homomorphism from the natural projection $\iota_{p}:\{p\} \rightarrow M_{\alpha}$. We define the set

$$
Z(\xi):=\left\{p \in M_{\alpha}^{T}|\xi|_{p}=0\right\} .
$$

We call the cardinality of $Z(\xi)$ the zero-length of $\xi$, denote it $|Z(\xi)|$. The following proposition tells us that the zero-length $|Z(\xi)|$ is invariant under an algebra isomorphism.

Proposition 8.5. Let $f_{T}^{*}: H_{T}^{*}\left(M_{\alpha}\right) \rightarrow H_{T}^{*}\left(M_{\beta}\right)$ be a weak $H^{*}(B T)$-algebra isomorphism. Then, the following equation holds for all $\xi \in H_{T}^{*}\left(M_{\alpha}\right)$ :

$$
|Z(\xi)|=\left|Z\left(f_{T}^{*}(\xi)\right)\right| \text {. }
$$
$S$, i.e.,

Proof. Let $S=H^{*}(B T) \backslash\{0\}$ and let $S^{-1} H_{T}^{*}(M)$ denote the localized ring of $H_{T}^{*}(M)$ by

$$
S^{-1} H_{T}^{*}(M)=\left\{\frac{r}{s} \mid r \in H_{T}^{*}(M), s \in S\right\} / \sim
$$

where

$$
\frac{r_{1}}{s_{1}} \sim \frac{r_{2}}{s_{2}} \Longleftrightarrow\left(r_{1} s_{2}-r_{2} s_{1}\right) t=0 \text { for some } t \in S \text {. }
$$

Due to Theorem 5.4 and Proposition $6.1, H_{T}^{*}\left(M_{\alpha} ; \mathbb{Z}\right)$ is free as a module over $H^{*}(B T ; \mathbb{Z})$. Hence, because of the localization theorem in equivariant cohomology (see [Hs, p.40]), the natural map

$$
H_{T}^{*}\left(M_{\alpha}\right) \longrightarrow S^{-1} H_{T}^{*}\left(M_{\alpha}\right) \simeq S^{-1} H_{T}^{*}\left(M_{\alpha}^{T}\right)=\bigoplus_{p \in M_{\alpha}^{T}} S^{-1} H_{T}^{*}(\{p\})
$$


is injective (also see [HaPr, Theorem 2.5]), where the isomorphism appearing in the above maps is induced from the inclusion map from $M_{\alpha}^{T}$ to $M_{\alpha}$. Therefore, we may regard an element $\xi \in$ $H_{T}^{*}\left(M_{\alpha}\right)$ as an element in $S^{-1} H_{T}^{*}\left(M_{\alpha}\right)$. Then, the annihilator

$$
\operatorname{Ann}(\xi):=\left\{\eta \in S^{-1} H_{T}^{*}\left(M_{\alpha}\right) \mid \eta \xi=0\right\} \subset \bigoplus_{p \in M_{\alpha}^{T}} S^{-1} H_{T}^{*}(\{p\})
$$

of $\xi$ is nothing but the sum of $S^{-1} H_{T}^{*}(\{p\})$ over $p$ with $\left.\xi\right|_{p}=0$, because $\left.\eta\right|_{p}=0$ if $\left.\xi\right|_{p} \neq 0$. Therefore, $\operatorname{Ann}(\xi)$ is a free $S^{-1} H^{*}(B T)$-module of rank $|Z(\xi)|$. Since $f_{T}^{*}$ is a weak $H^{*}(B T)$ algebra isomorphism, we have

$$
\operatorname{Ann}(\xi) \simeq \operatorname{Ann}\left(f_{T}^{*}(\xi)\right)
$$

as a free $S^{-1} H^{*}(B T)$-module. This implies that $|Z(\xi)|=\left|Z\left(f_{T}^{*}(\xi)\right)\right|$.

Put $\mathcal{T}^{(1)}$ and $\mathcal{T}^{(2)}$ be the canonical generators in $H_{T}^{*}\left(M_{\alpha}\right)$ and $H_{T}^{*}\left(M_{\beta}\right)$, respectively. Let $\mathcal{T}_{0}^{(s)} \subset \mathcal{T}^{(s)}$, for $s=1,2$, be the canonical generators whose zero-length are zero. Let $\mathcal{T}_{1}^{(s)}$ be the set in $\mathcal{T}^{(s)} \backslash \mathcal{T}_{0}^{(s)}$ with largest zero-length, and let $\mathcal{T}_{2}^{(s)}$ be the set in $\mathcal{T}^{(s)} \backslash \mathcal{T}_{0}^{(s)}$ second largest zero-length, and so on.

REMARK 8.6. In toric manifolds, all of canonical generators satisfy $\left|Z\left(\tau_{i}\right)\right| \neq 0$, i.e., $\mathcal{T}_{0}^{(s)}=\emptyset$. However, in toric hyperKähler manifolds, there exists a canonical generator $\tau_{i}$ such that $\left|Z\left(\tau_{i}\right)\right|=$ 0 , i.e., $\mathcal{T}_{0}^{(s)} \neq \emptyset$. For example, in the equivariant cohomology of a toric hyperKähler manifold $M=M^{\prime} \times \mathbb{H}$, the canonical generator $\tau$ which corresponds to the characteristic submanifold $M^{\prime}$ satisfies $|Z(\tau)|=0$ because $M^{T}=\left(M^{\prime}\right)^{T}$. This gives one of the difference between the proof of the Masuda's theorem (see Theorem 1 in Section 1) for toric manifolds proved in [Ma2] and that of Theorem 1.1 in Section 1 for toric hyperKähler manifolds.

We first show the following property for $\mathcal{T}_{0}^{(s)}(s=1,2)$ :

Proposition 8.7. If there exists $\tau_{i} \in \mathcal{T}_{0}^{(s)}(s=1,2)$, then there exists the element $x_{i} \in$ $H^{2}(B T)$ such that

$$
\pi_{s}^{*}\left(x_{i}\right)=\tau_{i}
$$

Proof. Assume $\tau_{i} \in \mathcal{T}_{0}^{(1)}$. By definition, we have that $\left.\tau_{i}\right|_{p} \neq 0$ for all $p \in M^{T}$. Recall that the canonical generator $\tau_{i}$ corresponds to the characteristic submanifold $M_{i}$, and $M_{i}$ corresponds to the hyperplane $H_{i} \subset H^{2}\left(B T ; \mathbb{R}\right.$ ) (see Section 5.2). Using $\left.\tau_{i}\right|_{p} \neq 0$ for all $p \in M^{T}$, it is easy to check that $\left(M_{i}\right)^{T}=M_{\alpha}^{T}$. Therefore, because fixed points correspond to the intersection of just $n$ hyperplanes, we have that $H_{i} \cap H_{j} \neq \emptyset$ for all $j \neq i$. Because there are no intersection points of just $n$ hyperplanes in the complement of $H_{i}\left(\subset H^{2}(B T ; \mathbb{R})\right)$, there exists a primitive $x_{i} \in H^{2}(B T)$ such that all of the hyperplanes $H_{j}($ where $j \neq i)$ satisfy

$$
H_{j}=L_{j} \times \mathbb{R} x_{i},
$$

where $L_{j}=H_{i} \cap H_{j}$ is the $(n-2)$-dimensional hyperplane (see Figure 4$)$. This means that, for all $x \in H_{j}(j \neq i)$, there exists $x^{\prime} \in L_{j}=H_{i} \cap H_{j}$ and $r \in \mathbb{R}$ such that

$$
x=x^{\prime}+r x_{i} .
$$

Hence, we have the following relations:

$$
\begin{aligned}
0 & =\left\langle\pi_{1}^{*}(x)+\widehat{a}, \mathbf{u}_{j}\right\rangle_{H} & & \left(\text { by } x \in H_{j}\right) \\
& =\left\langle\pi_{1}^{*}\left(x^{\prime}+r x_{i}\right)+\widehat{a}, \mathbf{u}_{j}\right\rangle_{H} & & \left(\text { by } x=x^{\prime}+r x_{i}\right) \\
& =\left\langle\pi_{1}^{*}\left(r x_{i}\right), \mathbf{u}_{j}\right\rangle_{H} & & \left(\text { by } x^{\prime} \in H_{j}\right) .
\end{aligned}
$$

Therefore, we have that

$$
\left\langle\pi_{1}^{*}\left(x_{i}\right), \mathbf{u}_{j}\right\rangle_{H}=0 \text { for all } j \neq i .
$$

Because $\pi_{1}^{*}$ is injective, this also implies that $\left\langle\pi_{1}^{*}\left(x_{i}\right), \mathbf{u}_{i}\right\rangle \neq 0$. Because $\tau_{i}$ is the dual basis of $\mathbf{u}_{i}$ (see Section 7.2), we have that

$$
\pi_{1}^{*}\left(x_{i}\right)=r^{\prime} \tau_{i} \in H_{T}^{2}\left(M_{\alpha}\right)
$$




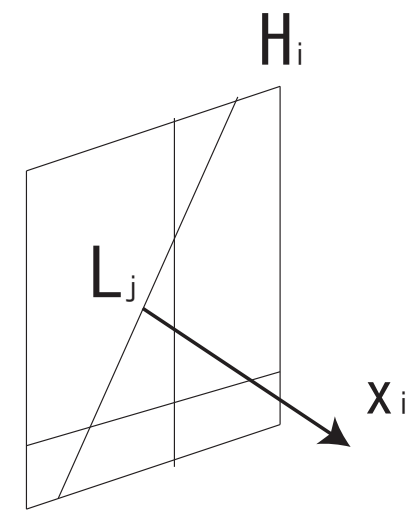

Figure 4 . The hyperplane $H_{j}=L_{j} \times \mathbb{R} x_{i}$. Three lines in the hyperplane $H_{i}$ represent the intersections with the other hyperplanes and $H_{j} \cap H_{i}=L_{i}$.

for $r^{\prime}=\left\langle\pi_{1}^{*}\left(x_{i}\right), \mathbf{u}_{i}\right\rangle \in \mathbb{Z} \backslash\{0\}$. Therefore, one can easily show that

$$
\left.r^{\prime} \tau_{i}\right|_{p}=x_{i}
$$

for all $p \in M_{\alpha}^{T}$ (e.g. by using the localization theorem, also see [HaHo, HaPr]). Because $x_{i}$ is a primitive vector in $H^{2}(B T)$, we have that $r^{\prime}= \pm 1$. Therefore, by changing the sign if we need, we have that there exists $x_{i} \in H^{2}(B T)$ such that $\pi_{1}^{*}\left(x_{i}\right)=\tau_{i}$. This establishes the statement for $s=1$.

Similarly, we have the statement for $s=2$.

Next, we prove that $f_{T}^{*}$ preserves $\mathcal{T}_{k}^{(1)}$ to $\mathcal{T}_{k}^{(2)}$ for $k \neq 0$ (see Lemma 8.9). In order to do that, we need the following lemma:

LEMMA 8.8. Let $\xi \in H_{T}^{2}\left(M_{\alpha}\right)$ be an element with $|Z(\xi)| \neq 0$. Express $\xi=\sum_{i=1}^{m} a_{i} \tau_{i}$ for some integers $a_{i}$. If $a_{i} \neq 0$ for some $i$, then $Z(\xi) \subset Z\left(\tau_{i}\right)$ for $\tau_{i} \in \mathcal{T}^{(1)} \backslash \mathcal{T}_{0}^{(1)}$.

Furthermore, if $a_{i} \neq 0$ and $a_{j} \neq 0$ for some different $i$ and $j$, then $Z(\xi) \subsetneq Z\left(\tau_{i}\right)$ for $\tau_{i} \in$ $\mathcal{T}^{(1)} \backslash \mathcal{T}_{0}^{(1)}$

Proof. Let $p \in M_{\alpha}^{T}$ and $p \in Z(\xi)$. Then $0=\left.\xi\right|_{p}=\left.\sum_{i=1}^{m} a_{i} \tau_{i}\right|_{p}$. Using Corollary 7.5, we have that if $a_{i} \neq 0$ then $\left.\tau_{i}\right|_{p}=0$. This establishes that if $a_{i} \neq 0$ then $Z(\xi) \subset Z\left(\tau_{i}\right)$; moreover, if both $a_{i}$ and $a_{j}$ are non-zero, then $Z(\xi) \subset Z\left(\tau_{i}\right) \cap Z\left(\tau_{j}\right)$. Therefore, it suffices to prove that $Z\left(\tau_{i}\right) \cap Z\left(\tau_{j}\right)$ is properly contained in $Z\left(\tau_{i}\right)$.

Suppose that $Z\left(\tau_{i}\right) \cap Z\left(\tau_{j}\right)=Z\left(\tau_{i}\right)$. Then $Z\left(\tau_{j}\right) \supset Z\left(\tau_{i}\right)$. By (7.12), we have that

$$
\left.\tau_{i}\right|_{q}=0 \text { if and only if } q \notin M_{i}^{T} .
$$

Therefore, $M_{j}^{T} \subset M_{i}^{T}$. Recall that fixed points of $\left(M_{\alpha}, T\right)$ correspond to the intersections of just $n$ hyperplanes. Hence, the condition $M_{j}^{T} \subset M_{i}^{T}$ gives that the corresponding hyperplanes $H_{j}$ and $H_{i}$ satisfy that $H_{j} \cap H_{i}=L_{j}$ is the $(n-2)$-dimensional hyperplane and

$$
H_{j}=L_{j} \times \mathbb{R} x_{i}
$$

for some $x_{i}$; a normal vector of $H_{i}$ (also see the proof of Proposition 8.7). Because we assume $\left.\tau_{i}\right|_{p}=0$, there exists $x \notin H_{i} \cap H_{j}$ in $H^{2}(B T ; \mathbb{R})$ such that $\{x\}=\cap_{k \in I(p)} H_{k}$ by using (8.2) (also see Figure 5), where $I(p) \subset[m] \backslash\{i, j\}$ is the set satisfying that $\{p\}=\cap_{k \in I(p)} M_{k}$, i.e., $x \in H^{2}(B T ; \mathbb{R})$ is the corresponding intersection point to $p \in M_{\alpha}^{T}$. Because the hyperplane arrangement is smooth, it is easy to check that there exists $l \in I(p)$ such that, for $I^{\prime}(p)=I(p) \backslash\{l\}$, the intersection $\cap_{k \in I^{\prime}(p)} H_{k}$ is the 1-dimensional affine subspace which goes through $x$ and $H_{j}$. It also follows from $I(p) \subset[m] \backslash\{i, j\}$ that the 1-dimensional affine subspace $\cap_{k \in I^{\prime}(p)} H_{k}$ does not intersect with $L_{j}=H_{i} \cap H_{j}$. Therefore, there is the point $\{y\}=\left(\cap_{k \in I^{\prime}(p)} H_{k}\right) \cap H_{j}$ such that $y \notin H_{i}$ (see Figure 5). This gives a contradiction to the assumption that $M_{j}^{T} \subset M_{i}^{T}$. Therefore, $Z\left(\tau_{i}\right) \cap Z\left(\tau_{j}\right) \neq Z\left(\tau_{i}\right)$. 


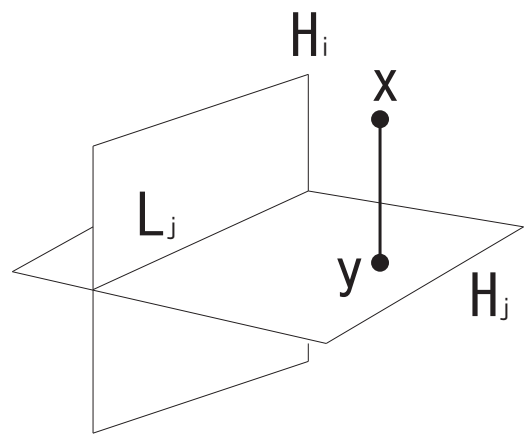

Figure 5

This establishes that $Z(\xi) \subsetneq Z\left(\tau_{i}\right)$.

Now we may prove Lemma 8.9.

LEMma 8.9. Every weak $H^{*}(B T)$-algebra isomorphism $f_{T}^{*}$ preserves $\mathcal{T}_{k}^{(1)}$ to $\mathcal{T}_{k}^{(2)}$ up to sign for $k \neq 0$.

Proof. Let $m_{k}^{(1)}$ (resp. $m_{k}^{(2)}$ ) be the zero-length of elements in $\mathcal{T}_{k}^{(1)}$ (resp. $\mathcal{T}_{k}^{(2)}$ ).

By using Lemma 8.8, if $\xi=\sum_{i=1}^{m} a_{i} \tau_{i}^{(1)}$ satisfies $a_{i}, a_{j} \neq 0$ then $|Z(\xi)|<\left|Z\left(\tau_{i}^{(1)}\right)\right|$. Therefore, we have that $m_{1}^{(1)}$ is the highest zero-length in $H_{T}^{2}\left(M_{\alpha}\right)$, and if $|Z(\xi)|=m_{1}^{(1)}$ then $\xi=a_{i} \tau_{i}^{(1)}$ for some non-zero integer $a_{i}$ and $\tau_{i}^{(1)} \in \mathcal{T}_{1}^{(1)}$. Similarly, we have that $m_{1}^{(2)}$ is the highest zero-length in $H_{T}^{2}\left(M_{\beta}\right)$, and if $|Z(\xi)|=m_{1}^{(2)}$ then $\xi=b_{i} \tau_{i}^{(2)}$ for some non-zero integer $b_{i}$ and $\tau_{i}^{(2)} \in \mathcal{T}_{1}^{(2)}$. Take an element $\tau_{i}^{(1)} \in \mathcal{T}_{1}^{(1)}$. Due to Proposition 8.5, $f_{T}^{*}\left(\tau_{i}^{(1)}\right)$ has the zero-length $m_{1}^{(1)}$. Moreover, $f_{T}^{*}\left(\tau_{i}^{(1)}\right)$ has the highest zero-length in $f_{T}^{*}\left(H_{T}^{2}\left(M_{\alpha}\right)\right)=H_{T}^{2}\left(M_{\beta}\right)$. Therefore, we have $m_{1}^{(1)}=m_{1}^{(2)}$. By using the arguments above, we have that $f_{T}^{*}\left(\tau_{i}^{(1)}\right)=b \tau_{j}^{(2)}$ for some $\tau_{j}^{(2)} \in \mathcal{T}_{1}^{(2)}$ and non-zero integer $b$. Because $f_{T}^{*}$ is isomorphism, we also have that $b= \pm 1$, i.e., $f_{T}^{*}$ maps $\mathcal{T}_{1}^{(1)}$ to $\mathcal{T}_{1}^{(2)}$ bijectively up to sign.

Take an element $\tau_{i}^{(1)} \in \mathcal{T}_{2}^{(1)}$. Because $\mathcal{T}_{1}^{(1)}$ and $\mathcal{T}_{1}^{(2)}$ are preserved under $f_{T}^{*}$ and $\left(f_{T}^{*}\right)^{-1}$, $f_{T}^{*}\left(\tau_{i}^{(1)}\right)$ does not have a term described by a linear combination of elements in $\mathcal{T}_{1}^{(2)}$. Therefore, by using Proposition 8.5 and Lemma 8.8, we have that $f_{T}^{*}\left(\tau_{i}^{(1)}\right)$ has the second highest zero-length $m_{2}^{(1)}$ in $f_{T}^{*}\left(H_{T}^{2}\left(M_{\alpha}\right)\right)=H_{T}^{2}\left(M_{\beta}\right)$. With the method similar to that demonstrated as above, we also have that $m_{2}^{(1)}=m_{2}^{(2)}$ and $f_{T}^{*}$ maps $\mathcal{T}_{2}^{(1)}$ to $\mathcal{T}_{2}^{(2)}$ bijectively up to sign. By repeating this argument, we have that $f_{T}^{*}$ preserves $\mathcal{T}_{k}^{(1)}$ to $\mathcal{T}_{k}^{(2)}$ up to sign for $k \neq 0$.

Let us prove Lemma 8.4 .

Proof of Lemma 8.4. Because of Lemma 8.9, $f_{T}^{*}$ preserves $\mathcal{T}^{(1)} \backslash \mathcal{T}_{0}^{(1)}$ to $\mathcal{T}^{(2)} \backslash \mathcal{T}_{0}^{(2)}$. Together with $\left|\mathcal{T}^{(1)}\right|=\left|\mathcal{T}^{(2)}\right|=m$, we may put

$$
\left|\mathcal{T}_{0}^{(1)}\right|=\left|\mathcal{T}_{0}^{(2)}\right|=m_{0} .
$$

Let $\mathcal{T}_{0}^{(s)}=\left\{\tau_{i}^{(s)} \mid i=1, \ldots, m_{0}\right\}$ for $s=1,2$. By Proposition 8.7, there exist elements $x_{i}, y_{i} \in$ $H^{2}(B T)$ such that $\pi_{1}^{*}\left(x_{i}\right)=\tau_{i}^{(1)}$ and $\pi_{2}^{*}\left(y_{i}\right)=\tau_{i}^{(2)}$ for $i=1, \ldots, m_{0}$. Therefore, by the exactness of the sequence (6.2), we have that $j^{*}\left(\tau_{i}^{(s)}\right)=0$. Hence, it follows from the assumption mentioned in Remark 2.3 that $a_{i}=b_{i}=0$, where $a_{i}, b_{i}$ are the $i^{\text {th }}$ coordinate of $\widehat{a}$ and $\widehat{b}$, respectively. Moreover, using Proposition 2.2, we have that $M_{\alpha}=M_{\alpha}^{\prime} \times \mathbb{H}^{m_{0}}$ and $M_{\beta}=M_{\beta}^{\prime} \times \mathbb{H}^{m_{0}}$ for some 
$4\left(n-m_{0}\right)$-dimensional toric hyperKähler manifolds $M_{\alpha}^{\prime}$ and $M_{\beta}^{\prime}$; therefore, we have

$$
\begin{aligned}
& H_{T}^{*}\left(M_{\alpha}\right) \simeq H_{T^{n-m_{0}}}^{*}\left(M_{\alpha}^{\prime}\right) \oplus H_{T^{m_{0}}}^{*}\left(\mathbb{H}^{m_{0}}\right), \\
& H_{T}^{*}\left(M_{\beta}\right) \simeq H_{T^{n-m_{0}}}^{*}\left(M_{\beta}^{\prime}\right) \oplus H_{T^{m_{0}}}^{*}\left(\mathbb{H}^{m_{0}}\right)
\end{aligned}
$$

such that $\mathcal{T}_{0}^{(s)}$ becomes the canonical generators in $H_{T^{m_{0}}}^{*}\left(\mathbb{H}^{m_{0}}\right)$ and $\mathcal{T}^{(1)} \backslash \mathcal{T}_{0}^{(1)}$ (resp. $\mathcal{T}^{(2)} \backslash \mathcal{T}_{0}^{(2)}$ ) becomes those in $H_{T^{n-m_{0}}}^{*}\left(M_{\alpha}^{\prime}\right)$ (resp. $H_{T^{n-m_{0}}}^{*}\left(M_{\beta}^{\prime}\right)$ ). Therefore, $f_{T}^{*}$ decomposes into

$$
f_{T}^{*}=f_{n-m_{0}}^{*} \oplus \varphi_{m_{0}}^{*} .
$$

where

$$
\varphi_{m_{0}}^{*}: H_{T^{m_{0}}}^{*}\left(\mathbb{H}^{m_{0}}\right) \rightarrow H_{T^{m_{0}}}^{*}\left(\mathbb{H}^{m_{0}}\right)
$$

is an isomorphism and

$$
f_{n-m_{0}}^{*}: H_{T^{n-m_{0}}}^{*}\left(M_{\alpha}^{\prime}\right) \rightarrow H_{T^{n-m_{0}}}^{*}\left(M_{\beta}^{\prime}\right)
$$

is a weak $H^{*}\left(B T^{n-m_{0}}\right)$-algebra isomorphism which preserves $\mathcal{T}^{(1)} \backslash \mathcal{T}_{0}^{(1)}$ to $\mathcal{T}^{(2)} \backslash \mathcal{T}_{0}^{(2)}$. Therefore, in order to prove Lemma 8.4, it is enough to change $\varphi_{m_{0}}^{*}$ into some isomorphism which preserves $\mathcal{T}_{0}^{(1)}$ to $\mathcal{T}_{0}^{(2)}$.

Because $\pi_{1}\left(x_{i}\right)=\tau_{i}^{(1)}$ and $\pi_{2}\left(y_{i}\right)=\tau_{i}^{(2)}$, we may identify $H^{*}\left(B T^{m_{0}}\right)$ and $H_{T^{m_{0}}}^{*}\left(\mathbb{H}^{m_{0}}\right)$ by $\pi_{s}$ and there exists a decomposition

$$
\varphi^{*}=\varphi_{n-m_{0}}^{*} \oplus \varphi_{m_{0}}^{*}
$$

for some isomorphism $\varphi_{n-m_{0}}^{*}: H^{*}\left(B T^{n-m_{0}}\right) \rightarrow H^{*}\left(B T^{n-m_{0}}\right)$ such that that the following diagram is commutative for each factor:

$$
\begin{gathered}
H^{2}\left(B T^{n-m_{0}}\right) \oplus H^{2}\left(B T^{m_{0}}\right) \stackrel{\pi_{1}^{*}}{\longrightarrow} H_{T^{n-m_{0}}}^{2}\left(M_{\alpha}^{\prime}\right) \oplus H_{T^{m_{0}}}^{2}\left(\mathbb{H}^{m_{0}}\right) \\
\downarrow \varphi^{*}=\varphi_{n-m_{0}}^{*} \oplus \varphi_{m_{0}}^{*} \\
H^{2}\left(B T^{n-m_{0}}\right) \oplus H^{2}\left(B T^{m_{0}}\right) \stackrel{\pi_{2}^{*}}{\longrightarrow} H_{T^{n-m_{0}}}^{2}\left(M_{\beta}^{\prime}\right) \oplus H_{T^{m_{0}}}^{2}\left(\mathbb{H}^{m_{0}}\right)
\end{gathered}
$$

i.e., $\varphi_{m_{0}}^{*} \circ \pi_{1}^{*}=\pi_{2}^{*} \circ \varphi_{m_{0}}^{*}$ and $f_{n-m_{0}}^{*} \circ \pi_{1}^{*}=\pi_{2}^{*} \circ \varphi_{n-m_{0}}^{*}$. Because $\varphi_{m_{0}}^{*}$ is an isomorphism and $\left\{x_{i}\right\}$ and $\left\{y_{i}\right\}$ are generators of $H^{*}\left(B T^{m_{0}}\right)$ (see the proof of Proposition 8.7), there exists an isomorphism $X: H^{*}\left(B T^{m_{0}}\right) \rightarrow H^{*}\left(B T^{m_{0}}\right)$ such that $X \circ \varphi_{m_{0}}^{*}\left(x_{i}\right)=y_{i}$. Then, it is easy to check that

$$
g_{T}^{*}=f_{n-m_{0}}^{*} \oplus X \circ \varphi_{m_{0}}^{*}
$$

is a weak $H^{*}(B T)$-algebra isomorphism which preserves $\mathcal{T}_{k}^{(1)}$ to $\mathcal{T}_{k}^{(2)}$ for $k \geq 0$. Moreover, $g_{T}^{*}(\widehat{a})=$ $\widehat{b}$ because $a_{i}=b_{i}=0$ for the coordinates in $H_{T^{m_{0}}}^{2}\left(\mathbb{H}^{m_{0}}\right)$. This establishes Lemma 8.4.

Now we may prove Proposition 8.3:

Proof of Proposition 8.3. Let $H_{i}^{(1)}$ and $H_{j}^{(2)}$ be hyperplanes in $\mathcal{H}_{\widehat{a}}$ and $\mathcal{H}_{\widehat{b}}$, respectively. Due to Lemma 8.4, there exists a weak $H^{*}(B T)$-algebra isomorphism $g_{T}^{*}$ such as Lemma 8.4. Because $g_{T}^{*}$ is a weak algebraic isomorphism, there exists an isomorphism $\varphi^{*}: H^{*}(B T) \rightarrow H^{*}(B T)$ such that $g_{T}^{*} \circ \pi_{1}^{*}=\pi_{2}^{*} \circ \varphi^{*}$. We claim $\varphi^{*}\left(H_{i}^{(1)}\right)=H_{\sigma(i)}^{(2)}$ for some permutation $\sigma:[m] \rightarrow[m]$. Because $g_{T}^{*}\left(\tau_{i}^{(1)}\right)=\epsilon_{i} \tau_{\sigma(i)}^{(2)}$, we have

$$
\left(g_{T}\right)_{*}\left(\mathbf{u}_{\sigma(i)}^{(2)}\right)=\epsilon_{i} \mathbf{u}_{i}^{(1)}
$$


for the dual basis $\mathbf{u}_{i}^{(1)} \in H_{2}^{T}\left(M_{\alpha}\right)$ and $\mathbf{u}_{\sigma(i)}^{(2)} \in H_{2}^{T}\left(M_{\beta}\right)$ of $\tau_{i}^{(1)}$ and $\tau_{\sigma(i)}^{(2)}$, respectively. Therefore, we have the following relations for $x \in H_{i}^{(1)}$ :

$$
\begin{aligned}
& \left\langle\pi_{2}^{*} \circ \varphi^{*}(x)+\widehat{b}, \mathbf{u}_{\sigma(i)}^{(2)}\right\rangle_{H} \\
& =\left\langle g_{T}^{*} \circ \pi_{1}^{*}(x)+g_{T}^{*}(\widehat{a}), \mathbf{u}_{\sigma(i)}^{(2)}\right\rangle_{H} \quad \text { (by assumptions) } \\
& =\left\langle\pi_{1}^{*}(x)+\widehat{a},\left(g_{T}\right)_{*}\left(\mathbf{u}_{\sigma(i)}^{(2)}\right)\right\rangle_{H} \quad \text { (by dual) } \\
& =\left\langle\pi_{1}^{*}(x)+\widehat{a}, \epsilon_{i} \mathbf{u}_{i}^{(1)}\right\rangle_{H} \quad \text { (by (8.3)) } \\
& =0 \text {. } \\
& \text { (by } x \in H_{i}^{(1)} \text { ) }
\end{aligned}
$$

It follows from the relations above that we can easily prove $\varphi^{*}\left(H_{i}^{(1)}\right)=H_{\sigma(i)}^{(2)}$ for all $i \in[m]$. This establishes Proposition 8.3.

By using Proposition 8.2 and 8.3, we have Theorem 8.1.

Because of Theorem 4.4 and 8.1, we have Theorem 1.1.

REMARK 8.10. According to [HaPr], there exists the residual $S^{1}$-action on toric hyperKähler manifolds. The similar statement with Theorem 8.1 also holds for the $T^{n} \times S^{1}$-action on toric hyperKähler manifolds. We omit the detail of this fact in the present paper. This fact will be proved for more general context in the future works (also see $[\mathbf{K u}]$ ). Moreover, the cohomology rings and dimensions determine the diffeomorphism types of toric hyperKähler manifolds. Namely, the set of $4 n$-dimensional toric hyperKähler manifolds satisfy the cohomological rigidity for all $n \in \mathbb{N}$. The detail of this fact will be appeared in somewhere soon.

\section{Acknowledgements}

Finally the author would like to express his gratitude to Yasuzo Nishimura for invaluable advises and comments. In particular, Nishimura pointed out to him Proposition 3.8. He also would like to thank Professors DongYoup Suh and Mikiya Masuda for providing him excellent circumstances to do research.

\section{References}

[AtBo] M. Atiyah, R. Bott, The moment map and equivariant cohomology, Topology, 23 (1984), 1-28.

[Bi] R. Bielawski, Complete hyperkähler $4 n$-manifolds with a local tri-Hamiltonian $\mathbb{R}^{n}$-action, Math. Ann., 314 (1999), 505-528.

[BiDa] R. Bielawski, A. Dancer, The geometry and topology of toric hyperKäler, Comm. Anal. Geom., 8 (2000), 727-759.

[Br] G.E. Bredon, Introduction to compact transformation groups, Academic Press, 1972.

[BuPa] V.M. Buchstaber, T.E. Panov, Torus Actions and Their Applications in Topology and Combinatorics, University Lecture, 24, Amer. Math. Soc., Providence, R.I., 2002.

[Fu] W. Fulton, An Introduction to Toric Varieties, Ann. of Math. Studies, 113, Princeton Univ. Press, Princeton, N. J., 1993.

[Go] R. Goto, On toric hyper-Kähler manifolds given by the hyper-Kähler quotient method, Infinite analysis, Part A, B (Kyoto, 1991), 317-338, Adv. Ser. Math. Phys., 16, World Sci. Publ., River Edge, NJ, 1992.

[GuSt] V. Guillemin, S. Sternberg, Supersymmetry and equivariant de Rham theory, Springer Berlin, 1999.

[HaHo] M. Harada, T. Holm, The equivariant cohomology of hypertoric varieties and their real loci, Comm. Anal. Geom., 13 (2005), 645-677.

[HaMa] A. Hattori, M. Masuda, Theory of Multi-fans, Osaka. J. Math., 40 (2003), 1-68.

[HaPr] M. Harada, N. Proudfoot, Properties of the residual circle action on a hypertoric variety, Pacific J. of Math., 214 (2004), 263-284.

[HaSt] T. Hausel, B. Sturmfels, Toric hyperkähler varieties, Doc. Math., 7 (2002), 495-534.

[Hi] F. Hirzebruch, Über eine Klasse von einfachzusammenhängenden komplexen Mannigfaltigkeiten, Math. Ann., 124 (1951) 77-86.

[Hs] W.Y. Hsiang, Cohomology Theory of Topological Transformation Groups, Ergeb. Math., 85, Springer-Verlag, Berlin, 1975.

[Ka] K. Kawakubo, The theory of transformation groups, Oxford Univ. Press, London, 1991.

[Ko1] H. Konno, Equivariant cohomology rings of toric hyperkahler manifolds, Quaternionic structures in mathematics and physics (Rome, 1999), 231-240 (electronic), Univ. Studi Roma "La Sapienza", Rome, 1999.

[Ko2] H. Konno, Cohomology rings of toric hyperKähler manifolds, Int. J. of Math., 11 (2000), 1001-1026. 
[Ko3] H. Konno, The geometry of toric hyperkahler varieties. Toric topology, Contemp. Math., 460 (2008), 241260.

[Ku] S. Kuroki, A topological definition of hypertoric manifolds and its equivariant cohomology, Trends in Mathematics - New Series Vol 12 No 1, 135-138 (2010).

[Ma1] M. Masuda, Unitary toric manifolds, multi-fans and equivariant index, Tohoku Math. J., 51 (1999), 237-265.

[Ma2] M. Masuda, Equivariant cohomology distinguishes toric manifolds, Adv. Math., 218 (2008), 2005-2012.

[MaSu] M. Masuda, D. Y. Suh, Classification problems of toric manifolds via topology, Proc. of Toric Topology, Contemp. Math., 460 (2008), 273-286.

[MiTo] M. Mimura, H. Toda, Topology of Lie Groups, I and II, Amer. Math. Soc., 1991.

[Od] T. Oda, Convex Bodies and Algebraic Geometry. An Introduction to the Theory of Toric Varieties, Ergeb. Math. Grenzgeb. (3), 15, Springer-Verlag, Berlin, 1988.

[Pr] N. Proudfoot, A survey of hypertoric geometry and topology, Proc. of Toric topology, Contemp. Math., 460 (2008), 323-338.

Department of Mathematical Sciences, KAist, Daejeon 305-701, R. Korea

E-mail address: kuroki@kaist.ac.kr 